\title{
Fiber orders and compact spaces of uncountable weight
} by

\author{
Antonio Avilés (Murcia) and Ondřej F. K. Kalenda (Praha)
}

\begin{abstract}
We study an order relation on the fibers of a continuous map and its application to the study of the structure of compact spaces of uncountable weight.
\end{abstract}

1. Introduction and main results. This work is motivated by the following general problem: Given two compact convex sets $K$ and $L$ (in some locally convex linear topological spaces), are $K$ and $L$ homeomorphic? When $K$ and $L$ are metrizable (that is, they have countable weight) the well known Keller theorem (cf. [7, 17]) implies that $K$ and $L$ are homeomorphic if and only if they have the same dimension. Thus, when restricting our attention to compact sets of countable weight, only one topological invariant has to be computed to answer our question: the dimension, ranging from 0 to $\omega$.

When we pass to the case when the weight is uncountable, the situation is not that simple. A number of usual topological invariants, like chain conditions, cardinal functions, functional-analytic properties, etc., can be used to identify many different types of compact convex sets. Just to recall an elementary example, we may compare an uncountable product of intervals $[0,1]^{\kappa} \subset \mathbb{R}^{\kappa}$ with the ball $B(\kappa)$ of the Hilbert space $\ell_{2}(\kappa)$ in the weak topology. In $B(\kappa)$ we may find an uncountable family of disjoint open sets but $[0,1]^{\kappa}$ has the countable chain condition. Another argument would be that $B(\kappa)$ cannot be homeomorphic to an uncountable product since it contains $G_{\delta}$-points (we will prove a much subtler fact: $B(\kappa)$ is not even homeomorphic to a finite product of compact spaces of uncountable weight).

Very often, however, the standard topological technology is not so helpful as in the case of $B(\kappa)$ and $[0,1]^{\kappa}$. An example is when we restrict our attention to weakly compact sets of the Hilbert space $\ell_{2}(\kappa)$. The first example of a nonmetrizable weakly compact convex set not homeomorphic to $B(\kappa)$ may

2000 Mathematics Subject Classification: Primary 54B35; Secondary 52A07.

Key words and phrases: convex compact set, irreducible ordered set, semilattice of quotients, space of probability measures. 
be traced back to constructions of Corson and Lindenstrauss $[8,16]$, who provided such a set in which all points are $G_{\delta}$. Such sets, however, cannot be symmetric and to the best of our knowledge, only recently has the first author [1] provided a first example of an absolutely convex weakly compact subset of $\ell_{2}(\kappa)$ of weight $\kappa$ which is not homeomorphic to $B(\kappa)$. This was done by proving that $B(\kappa)$ satisfies a certain chain condition of Ramsey type introduced by Bell [4] and constructing ad hoc a compact convex set failing that property.

Let us now provide some natural examples of compact convex sets, all of them indeed representable as weakly compact convex subsets of $\ell_{2}(\kappa)$, for which apparently the standard techniques from topology give us no clue whether they are homeomorphic to each other or not (the letter $\kappa$ always denotes an uncountable cardinal):

- The ball of the Hilbert space, $B(\kappa)=\left\{x \in \ell_{2}(\kappa):\|x\|_{2} \leq 1\right\}$.

- The space $P(A(\kappa))$ of Radon probability measures on $A(\kappa)=\kappa \cup\{\infty\}$, the one-point compactification of the discrete set $\kappa$.

- The spaces $P\left(A(\kappa)^{n}\right), 2 \leq n<\omega$.

- The spaces $P\left(\sigma_{n}(\kappa)\right)$ of probability measures on $\sigma_{n}(\kappa)=\left\{x \in\{0,1\}^{\kappa}\right.$ : $|\operatorname{supp}(x)| \leq n\}, 2 \leq n<\omega$.

- The finite and countable powers of the previous spaces.

We shall develop some new tools which will allow us to conclude that all these spaces are not homeomorphic to each other, with perhaps the exception of $B(\kappa)$ and $P(A(\kappa))$ for which our techniques do not enable us to determine whether they are homeomorphic or not. We also studied other examples, not embeddable into a Hilbert space, namely the compact sets $P\left([0, \kappa]^{n}\right)^{m}$ for $\kappa$ an uncountable regular cardinal and $n, m \in \mathbb{N}$. In addition, we will obtain other applications concerning the structure of these spaces, regarding the following two kinds of questions:

- Classification of the points of a compact space $K$, that is: for which points $x, y \in K$ does there exist a homeomorphism $f: K \rightarrow K$ such that $f(x)=y$ ?

- When can a compact space $K$ be homeomorphic to some power compact of the form $L^{n}$, or when can it be homeomorphic to a product of the form $L_{1} \times \cdots \times L_{n}$ ?

All these questions are addressed through the beautiful technique of Shchepin of inverse limits and the spectral theorem developed in [19] and [20]. We explain this in detail in Section 2, but roughly speaking, given a compact space $K$ of uncountable weight, this technique allows one to study the topological structure of $K$ by studying the continuous surjections $p: X \rightarrow Y$ for $X$ and $Y$ quotients of $K$ of countable weight. And here comes the key 
idea of our work, to study a certain preorder relation induced on the fibers of a continuous map:

Definition 1.1. Let $f: K \rightarrow L$ be a continuous map and $x \in L$. We define a preorder relation $\leq$ on the fiber $f^{-1}(x)$ by letting $s \leq t$ if and only if for every neighborhood $U$ of $s$ there exists a neighborhood $V$ of $t$ such that $f(V) \subset f(U)$.

In other words, $s \leq t$ if and only if

$\{f(U): U$ is a neighborhood of $s\} \subset\{f(V): V$ is a neighborhood of $t\}$, if and only if $f^{-1} f(U)$ is a neighborhood of $t$ for every neighborhood $U$ of $s$. We shall write $\mathbb{F}_{x}(f)=f^{-1}(x)$ for the fiber of $x$ endowed with the preorder $\leq$ (and also with its inherent topology, though we shall not use the topological structure here). We denote by $\mathbb{O}_{x}(f)=\mathbb{F}_{x}(f) / \sim$ the ordered set obtained by quotienting by the equivalence relation $t \sim s \Leftrightarrow t \leq s$ and $s \leq t$.

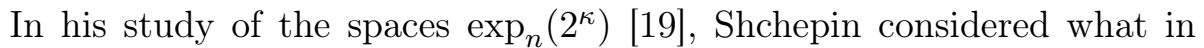
our language would be the cardinality of $\mathbb{O}_{x}(f)$. This was already useful in that discrete context but not in spaces like convex sets, where one needs to consider the order structure of $\mathbb{O}_{x}(f)$ to get some information.

Let us indicate how fiber orders may be helpful in the problem of classification of points of a compact space, and in the homeomorphic classification of compact sets. Consider a compact space $K$ of uncountable weight and a point $x \in K$. We can then consider the family of all fiber orders of type $\mathbb{O}_{p_{L}(x)}(q)$ for every continuous surjection $q: L^{\prime} \rightarrow L$ between metrizable quotients of $K$ with projections $p_{L^{\prime}}: K \rightarrow L^{\prime}, p_{L}: K \rightarrow L, q p_{L^{\prime}}=p_{L}$. This collection of ordered sets may be in principle rather complicated, but in the examples we deal with, almost all these sets are order isomorphic to the same ordered set that we can call $\mathbb{O}_{x}(K)$. For instance, for a finite power of the ball of the nonseparable Hilbert space $B(\kappa)$ we get the following picture:

Theorem 1.2. Let $K=B(\kappa)^{n}$ and $x=\left(x_{1}, \ldots, x_{n}\right) \in K$. Define $r=$ $\left|\left\{i:\left\|x_{i}\right\|<1\right\}\right|$. Then $\mathbb{O}_{x}(K) \cong[0,1]^{r}$.

We view $[0,1]^{r}$ as an ordered set endowed with the pointwise order, i.e.

$$
\left(t_{1}, \ldots, t_{r}\right) \leq\left(s_{1}, \ldots, s_{r}\right) \quad \text { iff } \quad t_{i} \leq s_{i} \text { for every } i .
$$

Let us record some consequences of this result other than the fact that the finite powers of the ball are nonhomeomorphic. It is a standard fact that the points of $B(\kappa)^{n}$ all of whose coordinates belong to the sphere are the $G_{\delta}$-points of $B(\kappa)^{n}$, and hence topologically different from the rest. We have obtained something much less evident: that points with different number of coordinates in the sphere are topologically different. This is a complete classification of the points of $B(\kappa)^{n}$ because if two points have the same 
number of coordinates in the sphere, then there is an automorphism of $B(\kappa)^{n}$ which moves one to the other.

Apart from the euclidean ball, we study spaces of probability measures on scattered spaces. We develop a general method for computing fiber orders in these cases, which constitutes the technically most involved part of our work. One of the key steps in this task is our Lemma 5.1 which probably has an independent interest. Every Radon probability measure on a scattered compact space is discrete, thus it is a certain (finite or infinite) convex combination of Dirac measures $\delta_{x}$. The following result (which follows immediately from Theorem 6.2 below) reduces the computation of fiber orders in $P(K)$ spaces to Dirac measures:

THEOREM 1.3. Let $K$ be a scattered compact and $\mu=\sum_{i \in I} r_{i} \delta_{x_{i}} \in$ $P(K)$, where $x_{i} \in K$ are pairwise distinct and $r_{i}>0$ for $i \in I$. Then

$$
\mathbb{O}_{\mu}(P(K)) \cong \prod_{i \in I} \mathbb{O}_{\delta_{x_{i}}}(P(K)) .
$$

The picture of the fiber orders of Dirac measures in our examples of probability measure spaces is the following:

Theorem 1.4. Let $K=\sigma_{n}(\kappa)$ and $x \in K$. Set $k=n-|x|$. Then $\mathbb{O}_{\delta_{x}}(P(K)) \cong\left\{\left(t_{1}, \ldots, t_{k}\right) \in[0,1]^{k}: t_{1} \leq \cdots \leq t_{k}\right\}$ where the order is defined as $t \leq s$ if and only if $t_{j} \leq s_{j}$ for every $j$.

In the next result, we denote by $2^{k}$ the power set of $\{1, \ldots, k\}$.

TheOREM 1.5. Let $K=A(\kappa)^{n}$ or $K=\left[0, \omega_{1}\right]^{n}$, and let $x=\left(x_{1}, \ldots, x_{n}\right)$ $\in K^{n}$. Set $k$ to be the number of coordinates of $x$ which are not $G_{\delta}$-points of $K$. Then $\mathbb{O}_{\delta_{x}}(P(K)) \cong\left\{\left(t_{A}\right)_{A \in 2^{k}} \in[0,1]^{2^{k}}: \sum_{A \in 2^{k}} t_{A}=1\right\}$ endowed with the order $\left(t_{A}\right) \leq\left(s_{A}\right)$ if and only if $\sum_{A \in \mathcal{A}} t_{A} \leq \sum_{A \in \mathcal{A}} s_{A}$ for every upwards closed family $\mathcal{A}$ of subsets of $\{1, \ldots, k\}$.

A similar statement holds for compact spaces $K=[0, \tau]$ with $\tau$ an uncountable regular cardinal, but for a modified version of the ordered sets $\mathbb{O}_{x}(L)$ relative to the cardinal $\tau$. Finally, we state results that we prove using decompositions of compact spaces as products:

Theorem 1.6. Let $K=B(\kappa), P\left(\sigma_{n}(\kappa)\right), P\left(A(\kappa)^{n}\right)$ or $P\left([0, \tau]^{n}\right)$ for $\tau$ an uncountable regular cardinal, and let $k, m \in \mathbb{N}$. Suppose that there exists a compact $L$ such that $K^{k} \approx L^{m}$. Then $k$ is a multiple of $m$.

Theorem 1.7. Let $K=B(\kappa), P(A(\kappa))$ or $P\left(\left[0, \omega_{1}\right]\right)$ and let $n, m \in \mathbb{N}$. Suppose that $L_{1}, \ldots, L_{m}$ are compact spaces of uncountable weight such that $K^{n} \approx \prod_{i=1}^{m} L_{i}$. Then $m \leq n$.

THEOREM 1.8. Let $\tau$ be a regular cardinal, $n, m$ natural numbers, and $L_{1}, \ldots, L_{m}$ compact spaces of weight $\tau$. If $P([0, \tau])^{n} \approx L_{1} \times \cdots \times L_{m}$, then $m \leq n$. 
We make two remarks about these results. First, our methods do not allow us to decide whether these compact spaces can be expressed as a nontrivial product with one factor metrizable. This appears not to be an easy question. Using a result of [21] and its variants, the second author [14] has shown that $P(K)$ is homeomorphic to $P(K) \times[0,1]$ for any compact scattered $K$. However, it is unknown to us whether $B(\kappa)$ is homeomorphic to $B(\kappa) \times[0,1]$. Second, the first author [3] has obtained with different techniques an improvement of Theorem 1.7: If $B(\kappa)^{n}$ maps continuously onto a product of nonmetrizable compacta of the form $\prod_{i=1}^{m} L_{i}$, then $m \leq n$. These techniques do not apply to the case of Theorem 1.6 for $K \neq B(\kappa)$, and actually $P\left(A(\kappa)^{n}\right)$ and $P\left(\sigma_{n}(\kappa)\right)$ map continuously onto $B(\kappa)^{n}$.

2. Spectral theory. In this section, we summarize in a self-contained way what we need about spectral theory, which is essentially taken from [19] and [20]. We also introduce the invariants $\mathbb{F}_{x}(K)$ and $\mathbb{O}_{x}(K)$, which play a central role in the paper.

Let $K$ be a compact space. We denote by $\mathcal{Q}(K)$ the set all Hausdorff quotient spaces of $K$, that is, the set all Hausdorff compact spaces of the form $K / E$ endowed with the quotient topology, for $E$ an equivalence relation on $K$. An element of $\mathcal{Q}(K)$ can be represented either by the equivalence relation $E$ or by the quotient space $L=K / E$ together with the canonical projection $p_{L}: K \rightarrow L$.

On the set $\mathcal{Q}(K)$ there is a natural order relation. In terms of equivalence relations, $E \leq E^{\prime}$ if and only if $E^{\prime} \subset E$. Equivalently, in terms of the quotient spaces, $L \leq L^{\prime}$ if and only if there is a continuous surjection $q: L^{\prime} \rightarrow L$ such that $q p_{L^{\prime}}=p_{L}$. The set $\mathcal{Q}(K)$ endowed with this order relation is a complete semilattice, that is, every subset has a least upper bound or supremum: if $\mathcal{E}$ is a family of equivalence relations of $\mathcal{Q}(K)$, its least upper bound is the relation given by $x E_{0} y$ if and only if $x E y$ for all $E \in \mathcal{E}$, in other words $E_{0}=\sup \mathcal{E}=\bigcap \mathcal{E}$. It is easy to check that $E_{0}$ gives a Hausdorff quotient if each element of $\mathcal{E}$ does.

Let $\mathcal{Q}_{\omega}(K) \subset \mathcal{Q}(K)$ be the family of all quotients of $K$ which have countable weight. Notice that $\sup \mathcal{A} \in \mathcal{Q}_{\omega}(K)$ for every countable subset $\mathcal{A} \subset \mathcal{Q}_{\omega}(K)$ and also that $\sup \mathcal{Q}_{\omega}(K)=K$. A family $\mathcal{S} \subset \mathcal{Q}_{\omega}(K)$ is called cofinal if for every $L \in \mathcal{Q}_{\omega}(K)$ there exists $L^{\prime} \in \mathcal{S}$ such that $L \leq L^{\prime}$. The family $\mathcal{S}$ is called a $\sigma$-semilattice if for every countable subset $\mathcal{A} \subset \mathcal{S}$, the least upper bound of $\mathcal{A}$ belongs to $\mathcal{S}$.

TheOREM 2.1 (A version of Shchepin's spectral theorem). Let $K$ be a compact space of uncountable weight and let $\mathcal{S}$ and $\mathcal{S}^{\prime}$ be two cofinal $\sigma$ semilattices in $\mathcal{Q}_{\omega}(K)$. Then $\mathcal{S} \cap \mathcal{S}^{\prime}$ is also a cofinal $\sigma$-semilattice in $\mathcal{Q}_{\omega}(K)$. 
Proof. The point is to prove that $\mathcal{S} \cap \mathcal{S}^{\prime}$ is cofinal. Let $L_{0} \in \mathcal{Q}_{\omega}(K)$ be arbitrary. Since $\mathcal{S}$ is cofinal there exists $L_{1} \in \mathcal{S}$ with $L_{0} \leq L_{1}$, similarly find $L_{2} \in \mathcal{S}^{\prime}$ with $L_{1} \leq L_{2}$, and continue by induction to obtain an increasing sequence with $L_{2 n+1} \in \mathcal{S}, L_{2 n} \in \mathcal{S}^{\prime}$. Finally, $L=\sup \left\{L_{n}: n<\omega\right\} \in \mathcal{S} \cap \mathcal{S}^{\prime}$ since both sets are $\sigma$-semilattices.

It may not be obvious how to check whether a given $\sigma$-semilattice is cofinal, so this theorem must be applied together with the following criterion:

Lemma 2.2. Let $K$ be a compact space of uncountable weight and $\mathcal{S}$ a $\sigma$-semilattice in $\mathcal{Q}_{\omega}(K)$. Then $\mathcal{S}$ is cofinal if and only if $\sup \mathcal{S}=K$.

Proof. If $\mathcal{S}$ is cofinal, then $\sup \mathcal{S}=\sup \mathcal{Q}_{\omega}(K)=K$. Conversely, suppose that $\sup \mathcal{S}=K$. Consider the family $\mathcal{A}$ of all continuous functions $f: K \rightarrow \mathbb{R}$ such that there exists $L \in \mathcal{S}$ such that $f$ factors through $p_{L}: K \rightarrow L$, that is, there exists $\hat{f}: L \rightarrow \mathbb{R}$ with $f=\hat{f} p_{L}$. As $\mathcal{S}$ is a $\sigma$-semilattice, $\mathcal{A}$ is a subalgebra of the algebra $C(K)$ of real-valued continuous functions on $K$. Clearly, the constant functions belong to $\mathcal{A}$ and $\operatorname{since} \sup \mathcal{S}=K, \mathcal{A}$ separates the points of $K$. Hence, by the Stone-Weierstrass theorem every $f \in C(K)$ is the limit of a sequence of functions from $\mathcal{A}$. But indeed $\mathcal{A}$ is closed under limits of sequences, namely if $f_{n}$ factors through $L_{n} \in \mathcal{S}$, then $\lim f_{n}$ factors through $\sup \left\{L_{n}: n<\omega\right\} \in \mathcal{S}$. We conclude that $\mathcal{A}=C(K)$. Now, if $p: K \rightarrow L$ is an arbitrary element of $\mathcal{Q}_{\omega}(K)$, then we can take an embedding $L \subset \mathbb{R}^{\omega}$ and consider the functions $e_{n} p: K \rightarrow \mathbb{R}$ obtained by composing with the coordinate functions $e_{n}: \mathbb{R}^{\omega} \rightarrow \mathbb{R}$. For every $n$, since $\mathcal{A}=C(K)$, there exists $L_{n} \in \mathcal{S}$ such that $e_{n} p$ factors through $L_{n}$. Finally, this implies that $p$ factors through $L_{\infty}=\sup \left\{L_{n}: n<\omega\right\}$, so $L \leq L_{\infty} \in \mathcal{S}$.

The importance of this machinery is that it allows us to study a compact space of uncountable weight by studying the cofinal $\sigma$-semilattice of metrizable quotients, and particularly the natural projections between elements of the $\sigma$-semilattice. In this way, the study of compact spaces of uncountable weight is linked to the study of continuous surjections between compact spaces of countable weight. The following language will be useful:

Definition 2.3. Let $K$ be a compact space of uncountable weight and let $\mathcal{P}$ be a property. We say that the $\sigma$-typical surjection of $K$ has property $\mathcal{P}$ if there exists a cofinal $\sigma$-semilattice $\mathcal{S} \subset \mathcal{Q}_{\omega}(K)$ such that for any elements $L<L^{\prime}$ of $\mathcal{S}$, the natural projection $p: L^{\prime} \rightarrow L$ has property $\mathcal{P}$.

It is a consequence of the spectral theorem that whether the $\sigma$-typical surjection of $K$ has a certain property can be checked on any given cofinal $\sigma$-semilattice:

THEOREM 2.4. Let $K$ be a compact space of uncountable weight, let $\mathcal{P}$ be a property, and let $\mathcal{S}$ be a fixed cofinal $\sigma$-semilattice in $\mathcal{Q}_{\omega}(K)$. Then the 
$\sigma$-typical surjection of $K$ has property $\mathcal{P}$ if and only if there exists a cofinal $\sigma$-semilattice $\mathcal{S}^{\prime} \subset \mathcal{S}$ such that for any elements $L<L^{\prime}$ of $\mathcal{S}^{\prime}$, the natural projection $p: L^{\prime} \rightarrow L$ has property $\mathcal{P}$.

The main kind of properties $\mathcal{P}$ that we shall be interested in concern the fiber orders of the surjections and the order relation that we defined on them. Given a point $x \in K$, we can study properties of the point $x$ by looking at the fiber order of $p_{L}(x)$ in the $\sigma$-typical $p: L^{\prime} \rightarrow L$. It may be useful to call this $\sigma$-typical fiber $\mathbb{F}_{x}(K)$, which we certainly cannot define as a concrete set, but rather as an abstract object, some properties of which we can predict.

Definition 2.5. Let $K$ be a compact space of uncountable weight and $x \in K$, and let $\mathcal{P}$ be a property. We say that $\mathbb{F}_{x}(K)$ has property $\mathcal{P}$ if $\mathbb{F}_{p_{L}(x)}(p)$ has property $\mathcal{P}$ for the $\sigma$-typical surjection $p: L^{\prime} \rightarrow L$.

In a similar way we shall talk about $\mathbb{O}_{x}(K)$. It is worth noticing that a point $x$ is a $G_{\delta}$-point of $K$ if and only if $\left|\mathbb{F}_{x}(K)\right|=1$. In other words, the information given by $\mathbb{F}_{x}(K)$ is trivial only when $x$ is a $G_{\delta}$-point of $K$. Namely, if $x$ is a $G_{\delta}$-point of $K$ then there is a continuous function $f: K \rightarrow[0,1]$ such that $x=f^{-1}(0)$. Then $f$ can be viewed as an element $L_{0} \in \mathcal{Q}_{\omega}(K)$ and we find that $\left|\mathbb{F}_{p_{L}(x)}(p)\right|=1$ for all $L^{\prime}>L>L_{0}$ and $p: L^{\prime} \rightarrow L$. Conversely, if $\left|\mathbb{F}_{x}(K)\right|=1$ then we can find $L \in \mathcal{Q}_{\omega}(K)$ such that $x=p_{L}^{-1}\left(p_{L}(x)\right)$. Another elementary example is the compact $K=L^{\kappa}$ where $L$ is a metrizable compact. In this case, one can show as an exercise that for every $x \in K$, $\mathbb{F}_{x}(K)$ is homeomorphic to $L^{\omega}$ and $\left|\mathbb{O}_{x}(K)\right|=1$.

3. Decomposition into products. In this section, apart from providing some basic facts that will be needed in the following, we prove two results, Theorems 3.7 and 3.9, which establish some sufficient conditions in terms of fiber orders in order that a compact $K$ cannot be decomposed as a product of other spaces in a certain way. In further sections, when computing the fiber orders of specific spaces, we will find that several compact spaces satisfy the assumptions of these results.

Definition 3.1. Let $P$ be a set and $\leq$ be a binary relation on $P$. We say that $(P, \leq)$ is a preordered set if

(1) $t \leq t$ for every $t \in P$,

(2) if $t \leq s$ and $s \leq u$, then $t \leq u$, for every $t, s, u \in P$.

If, moreover, for any $t, s \in P$, if $t \leq s$ and $s \leq t$ then $t=s$, then we say that $(P, \leq)$ is an ordered set. An ordered set $(O, \leq)$ is said to be linearly ordered if for any $t, s \in O$, either $t \leq s$ or $s \leq t$. 
There is a canonical way of constructing an ordered set from a given preordered set $(P, \leq)$, namely we consider the equivalence relation on $P$ given by $t \sim s$ iff $t \leq s$ and $s \leq t$, and then the quotient set $P / \sim$ is an ordered set when endowed with the relation induced from $P$. We call it the ordered set associated to $P$. When we write $p<q$ in a preordered set, it means that $p \leq q$ but $q \not \leq p$.

An order-isomorphism between the preordered sets $P$ and $Q$ is a bijection $f: P \rightarrow Q$ such that $f(t) \leq f(s)$ if and only if $t \leq s$.

Definition 3.2. Let $\left\{Q_{i}: i \in I\right\}$ be a family of preordered sets. The product of this family is the preordered set whose underlying set is the cartesian product $\prod_{i \in I} Q_{i}$ endowed by the preorder relation given by: $\left(t_{i}\right)_{i \in I} \leq$ $\left(s_{i}\right)_{i \in I}$ if and only if $t_{i} \leq s_{i}$ for every $i \in I$.

The product of an empty family of preordered sets is considered to be a singleton, with its only possible preorder structure. Products of preordered sets arise naturally in the context of fiber orders at least in two different situations, involving probability measures (cf. Theorem 6.2) and products of compact spaces:

Proposition 3.3. Let $\left\{f_{i}: K_{i} \rightarrow L_{i}: i \in I\right\}$ be a family of continuous surjections, let $f: \prod_{i \in I} K_{i} \rightarrow \prod_{i \in I} L_{i}$ be its product, and let $x=$ $\left(x_{i}\right)_{i \in I}$ be a point of $\prod_{i \in I} L_{i}$. Then the natural map $\mathbb{F}_{x}(f) \rightarrow \prod_{i \in I} \mathbb{F}_{x_{i}}\left(f_{i}\right)$ is an order-isomorphism. In particular, $\mathbb{F}_{x}(f) \cong \prod_{i \in I} \mathbb{F}_{x_{i}}\left(f_{i}\right)$ and $\mathbb{O}_{x}(f) \cong$ $\prod_{i \in I} \mathbb{O}_{x_{i}}\left(f_{i}\right)$.

The proof of this statement is straightforward. If $K$ is a finite or countable product of compact spaces, then a cofinal $\sigma$-semilattice in $\mathcal{Q}_{\omega}(K)$ is formed by all quotients of countable weight of $K$ which can be expressed as the product of a quotient of every factor. In this way, we see that the fibers of the $\sigma$-typical surjection of the product are the products of the fibers of the $\sigma$-typical surjection of every factor. We are thus allowed to write expressions like for instance $\mathbb{F}_{(x, y)}(K \times L) \cong \mathbb{F}_{x}(K) \times \mathbb{F}_{y}(L)$ or $\mathbb{F}_{\left(x_{1}, x_{2}, \ldots\right)}\left(\prod_{n<\omega} K_{n}\right) \cong$ $\prod_{n<\omega} \mathbb{F}_{x_{n}}\left(K_{n}\right)$.

Definition 3.4. An ordered set $O$ is called irreducible if whenever $O$ is isomorphic to a product $Q \times R$ then either $Q$ or $R$ is a singleton.

An elementary example of an irreducible ordered set is a linearly ordered set. An ordered set $O$ is called connected if whenever it is expressed as the disjoint union of two nonempty subsets $O=A \cup B$, there exist $a \in A$ and $b \in B$ such that either $a \leq b$ or $b \leq a$. All the ordered sets that appear in this note happen to be connected since indeed they have a minimum. The following Theorem 3.5 and its Corollary 3.6 are due to Hashimoto [12] and assert that any two decompositions of a connected ordered set as product have a common refinement, and consequently, a decomposition of a connected 
ordered set as a product of irreducible ordered sets is unique. Among other applications, this is a useful criterion to decide immediately that two given ordered sets are not isomorphic.

Theorem 3.5. Let $\mathcal{O}$ be a connected ordered set, and $\left\{O_{i}: i \in I\right\}$ and $\left\{Q_{j}: j \in J\right\}$ two families of ordered sets such that $O \cong \prod_{i \in I} O_{i} \cong \prod_{j \in J} Q_{j}$. Then there is a further family $\left\{Z_{i j}:(i, j) \in I \times J\right\}$ such that $O_{i} \cong \prod_{j \in J} Z_{i j}$ for every $i \in I$, and $Q_{j} \cong \prod_{i \in I} Z_{i j}$ for every $j \in J$.

Corollary 3.6. Let $\mathcal{O}$ be a connected ordered set, $\left\{O_{i}: i \in I\right\}$ a family of irreducible ordered sets and $\left\{Q_{j}: j \in J\right\}$ a family of arbitrary ordered sets. Assume that $O \cong \prod_{i \in I} O_{i} \cong \prod_{j \in J} Q_{j}$. Then there is a partition $I=\bigcup_{j \in J} F_{j}$ such that $Q_{j} \cong \prod_{i \in I_{j}} O_{i}$ for every $j \in J$.

We shall use the following terminology: Two continuous maps $f: U \rightarrow V$ and $f^{\prime}: U^{\prime} \rightarrow V^{\prime}$ are said to be homeomorphic if there exist homeomorphisms $u: U \rightarrow U^{\prime}$ and $v: V \rightarrow V^{\prime}$ such that $v f=f^{\prime} u$.

THEOREM 3.7. Let $K$ be a compact space of uncountable weight and let $O$ be a connected irreducible ordered set. Assume that there is $x \in K$ such that $\mathbb{O}_{x}(K) \cong O$ and that $\mathbb{O}_{y}(K) \neq O^{k}$ for each $y \in K$ and each $k>1$. If $K^{n} \approx L^{m}$ for some natural numbers $n, m$ and some space $L$, then $n$ is a multiple of $m$.

REMARK 3.8. Note that the assertion $\mathbb{O}_{y}(K) \nRightarrow O^{k}$ is not the negation of $\mathbb{O}_{y}(K) \cong O^{k}$. It rather means that for the $\sigma$-typical surjection $p$ we have $\mathbb{O}_{y}(p) \neq O^{k}$ (another remark about notation: we write $\mathbb{O}_{y}(p)=\mathbb{O}_{y^{\prime}}(p)$, where $y^{\prime}$ is the projection of $y$ on the range of $p$ ).

Proof of Theorem 3.7. Along this proof, it is important to have in mind that if $\mathcal{S}$ is a cofinal $\sigma$-semilattice in $\mathcal{Q}_{\omega}(X)$, then $\mathcal{S}^{k}=\left\{p_{Z}^{k}: X^{k} \rightarrow Z^{k}: Z \in \mathcal{S}\right\}$ is a cofinal $\sigma$-semilattice in $\mathcal{Q}_{\omega}\left(X^{k}\right), k \leq \omega$. Suppose that $n$ is not a multiple of $m$ and that $K^{n} \approx L^{m}$. Choose $x \in K$ with $\mathbb{O}_{x}(K) \cong O$. By Proposition 3.3 we get $\mathbb{O}_{(x, \ldots, x)}\left(K^{n}\right) \cong O^{n}$. Let $w=\left(w_{1}, \ldots, w_{m}\right) \in L^{m}$ correspond to $(x, \ldots, x)$ under this homeomorphism. Then, of course, $\mathbb{O}_{w}\left(L^{m}\right) \cong O^{n}$. Further, by Proposition 3.3, for the $\sigma$-typical surjection $q$ of $L$,

$$
O^{n} \cong \mathbb{O}_{w}\left(q^{m}\right) \cong \prod_{i=1}^{m} \mathbb{O}_{w_{i}}(q) .
$$

Using Corollary 3.6 and the fact that $n$ is not a multiple of $m$, we find that for the $\sigma$-typical surjection $q$ of $L$ there are $k \in\{1, \ldots, m\}$ and $n / m<s \leq n$ such that $\mathbb{O}_{w_{k}}(q) \cong O^{s}$. It follows that there are $k \in\{1, \ldots, m\}$ and $n / m<s \leq n$ such that in each cofinal $\sigma$-semilattice in $L$ there is some surjection $q$ with $\mathbb{O}_{w_{k}}(q) \cong O^{s}$ (this follows from Theorem 2.1: if not, for any $k, s$ there would be a corresponding cofinal $\sigma$-semilattice $\mathcal{S}_{k, s}$, and then $\bigcap \mathcal{S}_{k, s}$ gives a contradiction). Set $\widetilde{w}=\left(w_{k}, \ldots, w_{k}\right)$ and let $y=\left(y_{1}, \ldots, y_{n}\right) \in K^{n}$ 
correspond to $\widetilde{w}$ under the homeomorphism. By our assumptions there is a cofinal $\sigma$-semilattice $\mathcal{T} \subset \mathcal{Q}_{\omega}(K)$ such that $\mathbb{O}_{p\left(y_{i}\right)}(p) \neq O^{j}$ for any surjection $p$ in $\mathcal{T}, i=1, \ldots, n$ and $j>1$. Consider the cofinal $\sigma$-lattice $\mathcal{U}=\left\{Z \in \mathcal{Q}_{\omega}(L): Z^{m} \in \mathcal{T}^{n}\right\}$. By the previous argument, there exists a surjection $q$ in $\mathcal{U}$ such that $\mathbb{O}_{w_{k}}(q) \cong O^{s}$. The surjection $q^{m}$ corresponds to a surjection $p^{n}$ in $\mathcal{T}^{n}$ for which

$$
\prod_{i=1}^{n} \mathbb{O}_{y_{i}}(p) \cong \mathbb{O}_{y}\left(p^{n}\right) \cong O^{s m}
$$

As $s m>n$, Corollary 3.6 yields $\mathbb{O}_{p\left(y_{i}\right)}(p) \cong O^{j}$ for some $i \in\{1, \ldots, n\}$ and some $j>1$, a contradiction.

THEOREM 3.9. Let $K$ be a compact space of uncountable weight, $n$ a natural number, and $O$ a connected irreducible ordered set with $|O|>1$. Assume that the $\sigma$-typical surjection of $K, p: X \rightarrow Y$, has the following properties:

(1) For every $y \in Y, \mathbb{O}_{y}(p)$ is a connected ordered set.

(2) There is no $y \in Y$ with $\mathbb{O}_{y}(p) \cong O \times P$ with $|P|>1$.

(3) There exists $x \in Y$ such that $\mathbb{O}_{x}(p) \cong O$.

(4) For any $x \in Y$ with $\mathbb{O}_{x}(p) \cong O$ the preordered set $\mathbb{F}_{x}(p)$ has an equivalence class which is a singleton.

Then, if $L_{1}, \ldots, L_{m}$ are compact spaces of uncountable weight such that $K^{n} \approx L_{1} \times \cdots \times L_{m}$, then $m \leq n$.

Proof. Let $\mathcal{S}$ be a cofinal $\sigma$-semilattice in $\mathcal{Q}_{\omega}(K)$ in which all the natural projections have properties (1) to (4). Let $\mathcal{S}^{n}$ be the cofinal $\sigma$-semilattice in $\mathcal{Q}_{\omega}\left(K^{n}\right)$, as defined in the proof of Theorem 3.7. Consider the cofinal $\sigma$-semilattice $\mathcal{T}$ in $\mathcal{Q}_{\omega}\left(L_{1} \times \cdots \times L_{m}\right)$ whose elements are the quotients of $L_{1} \times \cdots \times L_{m}$ which are products of quotients of each coordinate, that is, of the form

$$
q_{1} \times \cdots \times q_{m}: L_{1} \times \cdots \times L_{m} \rightarrow Z_{1} \times \cdots \times Z_{m}
$$

for $q_{i}: L_{i} \rightarrow Z_{i}$ in $\mathcal{Q}_{\omega}\left(L_{i}\right)$. Since $K^{n} \approx L_{1} \times \cdots \times L_{m}$, the $\sigma$-semilattices $\mathcal{S}^{n}$ and $\mathcal{T}$ can be viewed as cofinal $\sigma$-semilattices of metrizable quotients over the same compact, so by Theorem 2.1 they intersect in a further cofinal $\sigma$ semilattice, and in particular, we can find a natural projection $p^{n}: X^{n} \rightarrow Y^{n}$ in $\mathcal{S}^{n}$ and a natural projection $q=q_{1} \times \cdots \times q_{m}: Z_{1} \times \cdots \times Z_{m} \rightarrow W_{1} \times \cdots \times$ $W_{m}$ in $\mathcal{T}$ which are homeomorphic. Of course, we have enough freedom to choose them in such a way that $W_{i} \neq Z_{i}$ for every $i$. Consider a point $w=$ $\left(w_{1}, \ldots, w_{m}\right)$ in $W_{1} \times \cdots \times W_{m}$ which corresponds under the homeomorphism to a point $(x, x, \ldots) \in Y^{n}$ with $\mathbb{O}_{x}(p) \cong O$. Thus, $\prod_{r=1}^{m} \mathbb{O}_{w_{r}}\left(q_{r}\right) \cong O^{n}$. After reordering if necessary, by Corollary 3.6 we know that $\left|\mathbb{O}_{w_{r}}\left(q_{r}\right)\right|=1$ for $r>n$. 
Claim A. $\left|\mathbb{O}_{v}\left(q_{r}\right)\right|=1$ for every $v \in W_{r}$ and every $r>n$.

Proof of the claim. Suppose for instance that there exists $v \in W_{n+1}$ with $\left|\mathbb{O}_{v}\left(q_{n+1}\right)\right|>1$. Let $w^{\prime}=\left(w_{1}, \ldots, w_{n}, v, w_{n+2}, \ldots\right)$ and let $y=\left(y_{1}, \ldots, y_{n}\right)$ $\in Y^{n}$ correspond to $w^{\prime}$ under the homeomorphism. Then $\prod_{i=1}^{n} \mathbb{O}_{y_{i}}(p) \cong$ $\mathbb{O}_{w^{\prime}}(q) \cong O^{n} \times \mathbb{O}_{v}\left(q_{n+1}\right)$. Using Theorem 3.5 and the fact that $O$ is irreducible, we conclude that there must exist $i$ such that $\mathbb{O}_{y_{i}}(p)$ is isomorphic to a set of the form $O \times P$ with $|P|>1$, which is a contradiction.

Claim B. $\left|\mathbb{F}_{v}\left(q_{r}\right)\right|=1$ for every $v \in W_{r}$ and every $r>n$.

Proof of the claim. Suppose for instance that there exists $v \in W_{n+1}$ with $\left|\mathbb{F}_{v}\left(q_{n+1}\right)\right|>1$, let $w^{\prime}=\left(w_{1}, \ldots, w_{n}, v, w_{n+2}, \ldots\right)$, and let $y=\left(y_{1}, \ldots, y_{n}\right)$ $\in Y^{n}$ correspond to $w^{\prime}$ under the homeomorphism. We know by Claim A that $\left|\mathbb{O}_{v}\left(q_{n+1}\right)\right|=1$, which means that $\mathbb{F}_{v}\left(q_{n+1}\right)$ consists of one equivalence class which is not a singleton. By Proposition 3.3, this translates into the fact that $\mathbb{F}_{w^{\prime}}(q) \cong \prod_{i=1}^{n} \mathbb{F}_{y_{i}}(p)$ has no equivalence class which is a singleton, which further implies that for some $i, \mathbb{F}_{y_{i}}(p)$ has no such equivalence class. Moreover, $\prod_{i=1}^{n} \mathbb{O}_{y_{i}}(p) \cong \mathbb{O}_{w^{\prime}}(q) \cong O^{n}$, so by Corollary 3.6 and our hypothesis $(2), \mathbb{O}_{y_{i}}(p) \cong O$ for every $i$. In this way, we have found a contradiction with our hypothesis (4).

Finally, notice that $\left|\mathbb{F}_{v}\left(q_{r}\right)\right|=1$ simply means that $q_{r}$ is one-to-one for $r>n$, that is, $Z_{r}=W_{r}$. Since we have supposed that $Z_{r} \neq W_{r}$ for all $r$, we conclude that $m \leq n$.

REMARK 3.10. Note that the previous theorem cannot be formulated just using $\mathbb{O}_{x}(K)$ and $\mathbb{F}_{x}(K)$ (while Theorem 3.7 is formulated in this way). Indeed, if $L$ is first countable, then $\mathbb{F}_{x}(L)$ is a singleton for each $x \in L$. Therefore $K$ and $K \times L$ cannot be distinguished using just the objects $\mathbb{O}_{x}(K)$ and $\mathbb{F}_{x}(K)$ and there are first countable compact spaces of uncountable weight.

4. The ball of the Hilbert space, $P(A(\kappa))$ and $M(A(\kappa))$. In this section we shall compute $\mathbb{O}_{x}$ for the ball of the Hilbert space and its finite powers. In particular, we shall prove Theorem 1.2, and Theorems 1.6 and 1.7 for the case $B(\kappa)$. Recall that

$$
B(\kappa)=\left\{\left(x_{i}\right)_{i<\kappa} \in \mathbb{R}^{\kappa}: \sum_{i<\kappa}\left|x_{i}\right|^{2} \leq 1\right\},
$$

endowed with the weak topology of the Hilbert space $\ell_{2}(\kappa)$. The weak topology clearly coincides with the pointwise topology. We can identify this space by the obvious homeomorphism with

$$
B(\kappa) \approx\left\{\left(x_{i}\right)_{i<\kappa} \in \mathbb{R}^{\kappa}: \sum_{i<\kappa}\left|x_{i}\right| \leq 1\right\} \subset \mathbb{R}^{\kappa}
$$


with the pointwise topology. This compact is also homeomorphic to the ball of $\ell_{p}(\kappa)$ for $1<p<\infty$ in the weak topology and to the dual ball of $c_{0}(\kappa)$ in the weak* topology. It is to be noticed that all the results proved in this section hold true (with essentially identical proof) if we substitute the space $B(\kappa)$ by $P(A(\kappa)) \approx\left\{\left(x_{i}\right)_{i<\kappa} \in[0,1]^{\kappa}: \sum_{i<\kappa} x_{i} \leq 1\right\}$. The fiber orders of $P(A(\kappa))$ will be computed again as a particular case of our methods in spaces of probability measures. We shall also notice that $P(A(\kappa))$ is not homeomorphic to the dual unit ball of the Banach space of continuous functions $C(A(\kappa))$ in its weak* topology.

For a subset $M$ of $\kappa$, we consider $B(M)=\left\{\left(x_{i}\right)_{i \in M} \in \mathbb{R}^{M}: \sum_{i \in M}\left|x_{i}\right| \leq 1\right\}$, and for $M \subset N$ we have the natural projection $p_{M N}: B(M) \rightarrow B(N)$ given by $p\left(\left(x_{i}\right)_{i \in M}\right)=\left(x_{i}\right)_{i \in N}$. Thus every $B(M)$ can be seen as a quotient of $B(\kappa)$ through the projection $p_{\kappa M}: B(\kappa) \rightarrow B(M)$, and all quotients of this type for $M$ a countably infinite subset of $\kappa$ constitute a cofinal $\sigma$ semilattice of $\mathcal{Q}_{\omega}(B(\kappa))$, as follows easily from Lemma 2.2. Hence, the $\sigma$ typical surjection of $B(\kappa)$ is of the form $p_{M N}: B(M) \rightarrow B(N)$ and its fiber orders are computed in the following way:

Lemma 4.1. Let $p_{M N}: B(M) \rightarrow B(N)$ be as above, and let $x \in B(N)$ and $y^{1}, y^{2} \in p_{M N}^{-1}(x)$. Then

$$
y^{1} \leq y^{2} \quad \text { if and only if } \quad \sum_{i \in M \backslash N}\left|y_{i}^{1}\right| \leq \sum_{i \in M \backslash N}\left|y_{i}^{2}\right| .
$$

Proof. Set $M^{*}=M \backslash N$. Let $y \in p_{M N}^{-1}(x)$. A basic neighborhood of $y$ is of the form

$$
U=\left\{z \in B(M): z_{i} \in W_{i} \text { for } i \in F\right\}
$$

where $F$ is a finite subset of $M$ and $W_{i}$ is an open real interval containing $y_{i}$ for every $i \in F$. Let $a_{i}=\inf \left\{|t|: t \in W_{i}\right\}$ be the distance of the interval $W_{i}$ to 0 . Then the image of the above typical basic neighborhood $U$ under $p_{N M}$ is

$$
\begin{aligned}
& p_{M N}(U)=\left\{z \in B(N): z_{i} \in W_{i} \text { for } i \in F \cap N\right\} \\
& \quad \text { if } 0 \in W_{i} \text { for all } i \in F \cap M^{*} ; \\
& p_{M N}(U)=\left\{\begin{aligned}
& z \in B(N): z_{i} \in W_{i} \text { for } i \in F \cap N \\
&\text { and } \left.\sum_{i \in N}\left|z_{i}\right|<1-\sum_{i \in F \cap M^{*}} a_{i}\right\} \quad \text { otherwise. }
\end{aligned}\right.
\end{aligned}
$$

This means that:

- If $y_{i}=0$ for all $i \in M^{*}$, then the images of basic neighborhoods of $y$ are basic neighborhoods of $x$.

- Otherwise, the images of basic neighborhoods of $y$ are of the form $V \cap\left\{z: \sum_{i \in N}\left|z_{i}\right|<1-r\right\}$ where $V$ is a basic neighborhood of $x$ and $0 \leq r<\sum_{i \in M^{*}}\left|y_{i}\right|$. 
From this description, it is already clear that if $\sum_{i \in M^{*}}\left|y_{i}^{1}\right| \leq \sum_{i \in M^{*}}\left|y_{i}^{2}\right|$ then $y^{1} \leq y^{2}$. For the converse, it is enough to check that if $r<s<$ $1-\sum_{i \in N}\left|x_{i}\right|$ there is no neighborhood $V$ of $x$ such that $V \cap\left\{z: \sum_{i \in N}\left|z_{i}\right|<\right.$ $1-r\} \subseteq\left\{z: \sum_{i \in N}\left|z_{i}\right|<1-s\right\}$. This follows from the fact that $N$ is infinite: Suppose $V=\left\{z \in B(N): z_{i} \in W_{i}\right.$ for $\left.i \in F\right\}$ where $F$ is some finite subset of $N$ and $W_{i}$ are intervals; take $t$ with $1-s<t<1-r$ and $n \in N \backslash F$; consider $y$ which agrees with $x$ on $F, y_{n}=t-\sum_{i \in F}\left|x_{i}\right|$, and $y_{i}$ is 0 in all other coordinates. Then $1-s<t=\sum_{i \in N}\left|y_{i}\right|<1-r$ and $y \in V$, so $y \in V \cap\left\{z: \sum_{i \in N}\left|z_{i}\right|<1-r\right\} \backslash\left\{z: \sum_{i \in N}\left|z_{i}\right|<1-s\right\}$.

It follows from Lemma 4.1 that $\mathbb{O}_{x}\left(p_{M N}\right)$ is order-isomorphic to an interval $[a, b]$ if $\sum_{i \in N}\left|x_{i}\right|<1$, and $\left|\mathbb{O}_{x}\left(p_{M N}\right)\right|=1$ if $\sum_{i \in N}\left|x_{i}\right|=1$. From this, it is also clear that for $x \in B(\kappa), \mathbb{O}_{x}(B(\kappa)) \cong[0,1]$ if $\sum_{i<\kappa}\left|x_{i}\right|<1$, and $\left|\mathbb{O}_{x}(B(\kappa))\right|=1$ if $\sum_{i<\kappa}\left|x_{i}\right|=1$. Theorem 1.2 now follows immediately.

We notice that $B(\kappa)$ satisfies the hypotheses of both Theorem 3.7 and Theorem 3.9, with $O=[0,1]$. An equivalence class of every fiber of $p_{N M}$ is a singleton, namely the class of the minimum element, the one with $y_{i}=0$ for all $i \in M^{*}$. This yields the proof of the case $B(\kappa)$ of Theorems 1.6 and 1.7.

We stated in the introduction that whenever two points $x, y \in B(\kappa)^{n}$ have the same number of coordinates in the sphere then there is a homeomorphism $f: B(\kappa)^{n} \rightarrow B(\kappa)^{n}$ with $f(x)=y$. Let us indicate why. It is enough to consider the case $n=1$. If $\|x\|=\|y\|$ then we can find a linear isometry of the Hilbert space $\ell_{2}(\kappa)$ onto itself sending $x$ to $y$. Then it remains to find some automorphism of $B(\kappa)$ sending an element of norm $\lambda$ to some element of norm $\mu$ for every $\lambda, \mu \in[0,1)$. View now again $B(\kappa)=$ $\left\{\left(x_{i}\right)_{i<\kappa}: \sum\left|x_{i}\right| \leq 1\right\}$. By the standard homeomorphism, the norm function is transformed into $\|x\|^{2}=\sum\left|x_{i}\right|$. Consider an increasing homeomorphism $\phi:[-1,1] \rightarrow[-1,1]$ such that $\phi(\lambda)=\mu$ and there exist one-sided derivatives which satisfy $\phi_{+}^{\prime}(-1)=1=\phi_{-}^{\prime}(1)$. Consider then $f: B(\kappa) \rightarrow B(\kappa)$ given by $f\left(\left(x_{i}\right)_{i<\kappa}\right)=\left(y_{i}\right)_{i<\kappa}$ where

$$
y_{0}=\phi\left(x_{0}\right) \quad \text { and } \quad y_{i}=\frac{1-\left|\phi\left(x_{0}\right)\right|}{1-\left|x_{0}\right|} x_{i} \quad \text { for } i>0 .
$$

Notice that $f$ is a homeomorphism and $f(\lambda, 0,0, \ldots)=(\mu, 0,0, \ldots)$.

Let $M(K)$ denote the set of all Radon measures of variation at most 1 (in other words, the dual ball of the Banach space $C(K)$ ) endowed with the weak* topology. We know that $\mathbb{O}_{x}(P(A(\kappa)))$ is either a singleton or orderisomorphic to $[0,1]$ for $x \in P(A(\kappa))$. A cofinal $\sigma$-semilattice for $\mathcal{Q}_{\omega}(M(K))$ is formed by the quotients of the form $M(p): M(K) \rightarrow M(L)$ where $p: K \rightarrow L$ is an element of $\mathcal{Q}_{\omega}(K)$, and hence the $\sigma$-typical surjection of $M(K)$ is of the form $M(g): M(X) \rightarrow M(Y)$, where $g: X \rightarrow Y$ is the $\sigma$-typical surjection of $K$. Hence, in order to prove that $P(A(\kappa)) \not \approx M(A(\kappa))$, it is enough to check the following: 
Proposition 4.2. Let $g: K \rightarrow L$ be a continuous surjection and let $f=M(g): M(K) \rightarrow M(L)$ be the induced map between the spaces of Radon measures of variation at most 1 . If there exists $x \in L$ such that $\left|\mathbb{O}_{x}(g)\right|>1$, then $\mathbb{O}_{0}(f)$ is not linearly ordered.

Proof. Take $y, z \in g^{-1}(x)$ such that $y \not \leq z$, so that there is a neighborhood $U$ of $y$ such that $g(U)$ does not contain the image of any neighborhood of $z$, and moreover $z \notin \bar{U}$. There is a net $\left(z_{\alpha}\right)$ in $K$ that converges to $z$ and with $g\left(z_{\alpha}\right) \notin g(U)$ for every $\alpha$. Consider the measures $\nu=\frac{1}{2} \delta_{y}-\frac{1}{2} \delta_{z}$ and $\mu=-\frac{1}{2} \delta_{y}+\frac{1}{2} \delta_{z}$. We claim that these are two incomparable elements of $f^{-1}(0)$. We prove that $\nu \not \leq \mu$ (that $\mu \not \leq \nu$ is proved by analogy). We consider $W=\left\{\lambda \in M(K): \lambda(U)>\frac{3}{8}\right\}$, which is a neighborhood of $\nu$. We claim that $f(W)$ does not contain the $f$-image of any neighborhood of $\mu$. Notice that $f(W) \subset\left\{\zeta \in M(L): \zeta(g(U))>-\frac{1}{4}\right\}$. Consider the measures $\mu_{\alpha}=-\frac{1}{2} \delta_{y}+\frac{1}{2} \delta_{z_{\alpha}}$. Then $\mu_{\alpha} \rightarrow \mu$ and $f\left(\mu_{\alpha}\right)(g(U))=-\frac{1}{2}$ for each $\alpha$. In particular, $f\left(\mu_{\alpha}\right) \notin f(W)$ for each $\alpha$. This witnesses that $\nu \not \leq \mu$.

\section{Computing images of neighborhoods in spaces of probability} measures. In order to compute the order of the fiber of a certain point $y \in K_{2}$ in a surjection $f: K_{1} \rightarrow K_{2}$, we have to know how to compute the images $f(U)$ of basic neighborhoods $U$ of points $x \in f^{-1}(y)$. The surjections which appear in the cases that we are going to study are of the form $f=$ $P(g): P(K) \rightarrow P(L)$ where $g: K \rightarrow L$ is a surjection between scattered compacta and $P$ is the functor of probability measure spaces. In this case, a neighborhood basis of a measure $\mu \in P(K)$ is formed by the sets of the form

$$
\mathcal{U}=\left\{\nu \in P(K): \nu\left(U_{i}\right)>c_{i}, i=1, \ldots, n\right\}
$$

where $U_{i}$ are disjoint clopen subsets of $K$ from a given basis of clopen sets, and $c_{i}$ are any numbers with $\mu\left(U_{i}\right)>c_{i}$. The following lemma determines the image $f(\mathcal{U})$ of such a neighborhood and will be applied repeatedly.

Lemma 5.1. Let $g: K \rightarrow L$ be a surjection between compact spaces, let $f=P(g): P(K) \rightarrow P(L), U_{1}, \ldots, U_{n}$ be disjoint closed subsets of $K$, $c_{1}, \ldots, c_{n} \geq 0$, and $\mathcal{U}=\left\{\nu \in P(K): \nu\left(U_{i}\right)>c_{i}, i=1, \ldots, n\right\}$. Then

$$
f(\mathcal{U})=\left\{\lambda \in P(L): \lambda\left(g\left(\bigcup_{i \in A} U_{i}\right)\right)>\sum_{i \in A} c_{i} \text { for } A \subset\{1, \ldots, n\}, A \neq \emptyset\right\} .
$$

The fact that $f(\mathcal{U})$ is included in the right-hand side expression is trivial. The other inclusion is related to the following numerical lemma:

Lemma 5.2. Let $c_{1}, \ldots, c_{n} \geq 0$, and $\alpha_{A} \geq 0$ for all $A \subset\{1, \ldots, n\}$, $A \neq \emptyset$, be numbers such that $\sum_{B \cap A \neq \emptyset} \alpha_{B}>\sum_{i \in A} c_{i}$ for every $A$. Then 
for each $A$ there exist numbers $\beta_{A, i}, i \in A$, such that $\sum_{i \in A} \beta_{A, i}=\alpha_{A}$ and moreover $\sum_{A \ni i} \beta_{A, i}>c_{i}$ for every $i=1, \ldots, n$.

Let us comment about the history of the lemmas. We first had a long inductive proof of Lemma 5.2. Then Richard Haydon indicated to us a more elegant and shorter proof using combinatorial optimization, which we reproduce below. Later, after David Fremlin heard about it at the Marczewski Centennial Conference in Będlewo, he wrote a note [10] where he showed that actually Lemma 5.1 holds under more general assumptions in $\mathcal{K}$-analytic spaces (our original statement was only for scattered or metrizable compact sets).

We first notice how Lemma 5.1 follows from Lemma 5.2 when $K$ is either scattered or metrizable, which is enough for the applications we present (for the general case we refer to [10]). Given a measure $\lambda$ on the right-hand side of the conclusion of Lemma 5.1, we consider $X_{A}=\bigcap_{i \in A} g\left(U_{i}\right) \backslash \bigcup_{i \notin A} g\left(U_{i}\right)$ and the numbers $\alpha_{A}=\lambda\left(X_{A}\right)$ (note that each $X_{A}$ is Borel as it is the difference of two closed sets), to which we can apply Lemma 5.2 and obtain the numbers $\beta_{A, i}$. We define a measure $\nu \in P(K)$ with $f(\nu)=\lambda$ in the following way.

Suppose first that $K$ and $L$ are scattered, so that all Radon measures on them are discrete, that is, determined by the measures of singletons (in this case, we do not even need the sets $U_{i}$ to be closed). If $y \in L \backslash \bigcup_{i=1}^{n} g\left(U_{i}\right)$ then we pick $x_{y} \in g^{-1}(y)$ and declare $\nu\left(\left\{x_{y}\right\}\right)=\lambda(\{y\})$. If $y \in X_{A}$ for some nonempty $A \subset\{1, \ldots, n\}$ with $\alpha_{A}>0$, then we can choose $x_{y, i} \in$ $f^{-1}(y) \cap U_{i}$ for every $i \in A$ and set $\nu\left(\left\{x_{y, i}\right\}\right)=\frac{\beta_{A, i}}{\alpha_{A}} \lambda(\{y\})$. At any other points, $\nu(\{x\})=0$. Then $\nu$ is a probability measure on $K$ with $f(\nu)=\lambda$ and moreover $\nu \in \mathcal{U}$ since $\nu\left(U_{i}\right)=\sum_{A \ni i} \beta_{A, i}$.

Now suppose that $K$ and $L$ are metrizable. Then Radon and Borel measures coincide. For each $A \subset\{1, \ldots, n\}$ and each $i \in A$, we set $Y_{A, i}=$ $g^{-1}\left(X_{A}\right) \cap U_{i}$, which is a nonempty Borel set. We also define $X_{\emptyset}=L \backslash$ $\bigcup_{i=1}^{n} g\left(U_{i}\right)$ and $Y_{\emptyset}=g^{-1}\left(X_{\emptyset}\right)$. By the Jankov-von Neumann Uniformization Theorem [15, Theorem 18.1], there exists a measurable selection $s_{A, i}: X_{A} \rightarrow$ $Y_{A, i}$ for the inverse of $g_{\mid Y_{A_{i}}}$ and also a measurable selection $s_{\emptyset}: X_{\emptyset} \rightarrow Y_{\emptyset}$ of the inverse of $g_{\mid Y_{\emptyset}}$. Consider

$$
\nu=s_{\emptyset}\left(\left.\lambda\right|_{X_{\emptyset}}\right)+\sum_{i \in A} \frac{\beta_{A, i}}{\alpha_{A}} s_{A, i}\left(\left.\lambda\right|_{X_{A, i}}\right) .
$$

Then $f(\nu)=\lambda$, and $\nu\left(U_{i}\right)=\sum_{A \ni i} \beta_{A, i}$, so $\nu \in \mathcal{U}$.

For the proof of Lemma 5.2, we shall use the so-called max-flow min-cut theorem from combinatorial optimization (Theorem 5.3 below). This result is originally due to Ford and Fulkerson [11] and Dantzig and Fulkerson [9], and can be found in the book [18, Theorem 10.3]. We have to recall some 
concepts from that area. A directed graph (digraph for short) is a couple $G=(V, A)$ where $V$ is a finite set whose elements are called vertices, and $A \subset V \times V$ is a set whose elements are called arcs. An $s$ - $t$ flow is a function $f: A \rightarrow(0,+\infty)$ which satisfies the flow conservation law at all points except $s$ and $t$ :

$$
\sum_{(x, u) \in A} f(x, u)=\sum_{(u, x) \in A} f(u, x) \quad \text { for every } u \in V \backslash\{s, t\} .
$$

In words, the flow entering $u$ equals the flow leaving $u$. The value of the flow $f$ is the net amount of flow leaving $s$, which happens to be equal to the net amount of flow entering $t$,

$$
\text { value }(f)=\sum_{(s, x) \in A} f(s, x)-\sum_{(x, s) \in A} f(x, s)=\sum_{(x, t) \in A} f(x, t)-\sum_{(t, x) \in A} f(t, x) .
$$

Let us a consider a function $c: A \rightarrow(0,+\infty)$ that we call a capacity function. A flow $f$ is said to be under $c$ if $f(u, v) \leq c(u, v)$ for every $(u, v) \in A$. Given a set $B \subset A$, the capacity of $B$ is $c(B)=\sum_{(u, v) \in B} c(u, v)$.

For a subset $U \subset V$, we denote by $\delta(U)$ the set of all arcs which leave $U$ and enter $V \backslash U$, that is,

$$
\delta(U)=\{(u, v) \in A: u \in U, v \notin U\} .
$$

For $s, t \in V$, an $s$ - $t$ cut is a set of arcs of the form $\delta(U)$, where $U \subset V$ with $s \in U$ and $t \notin U$.

Theorem 5.3 (max-flow min-cut theorem). Let $G=(V, A)$ be a digraph, $t, s \in V$ and $c: A \rightarrow \mathbb{R}_{+}$a capacity function. Then the maximum value of an $s$ - $t$ flow under $c$ equals the minimum capacity of an $s$ - $t$ cut, $\max \{\operatorname{value}(f): f \leq c$ is an $s$-t flow $\}=\min \{c(\delta(U)): U \subset V, s \in U, t \notin U\}$.

Proof of Lemma 5.2. We denote by $\mathcal{P}_{n}$ the family of all nonempty subsets of $\{1, \ldots, n\}$. First we consider numbers $c_{i}^{\prime}>c_{i}$ for every $i \leq n$ such that the inequalities

$$
\sum_{B \cap A \neq \emptyset} \alpha_{B}>\sum_{i \in A} c_{i}^{\prime}
$$

still hold. We consider a digraph $G=(V, A)$ where the set of vertices is

$$
V=\{s\} \cup\left\{p_{i}: 1 \leq i \leq n\right\} \cup\left\{q_{A}: A \in \mathcal{P}_{n}\right\} \cup\{t\},
$$

and the set of arcs is

$$
A=\left\{\left(s, p_{i}\right): 1 \leq i \leq n\right\} \cup\left\{\left(p_{i}, q_{A}\right): i \in A\right\} \cup\left\{\left(q_{A}, t\right): A \in \mathcal{P}_{n}\right\} .
$$

Let $M \in(0,+\infty)$ be such that $M>\sum_{i=1}^{n} c_{i}^{\prime}$. We define a capacity function $c: A \rightarrow \mathbb{R}_{+}$by

$$
c\left(s, p_{i}\right)=c_{i}^{\prime}, \quad c\left(p_{i}, q_{A}\right)=M, \quad c\left(p_{i}, q_{A}\right)=\alpha_{A} .
$$


Claim A. The minimal capacity of an s-t cut in $G$ equals $\sum_{i=1}^{n} c_{i}^{\prime}$.

Proof of Claim A. If $U=\{s\}$, then $c(\delta(U))=\sum_{i=1}^{n} c_{i}^{\prime}$. We suppose that $\delta(U)$ is an arbitrary $s$ - $t$ cut and we show that its capacity is larger than $\sum_{i=1}^{n} c_{i}^{\prime}$. Let $A=\left\{i \leq n: p_{i} \in U\right\}$. If there exists $B \in \mathcal{P}_{n}$ such that $q_{B} \notin U$ and $A \cap B \neq \emptyset$, then there exists $\left(p_{i}, q_{B}\right) \in \delta(U)$ and in particular $c(\delta(U)) \geq c\left(p_{i}, q_{B}\right)=M>\sum_{i=1}^{n} c_{i}^{\prime}$. Hence, we can suppose that $q_{B} \in U$ whenever $A \cap B \neq \emptyset$, therefore $\left(q_{B}, t\right) \in \delta(U)$ whenever $A \cap B \neq \emptyset$, and

$$
\begin{aligned}
c(\delta(U)) & \geq \sum_{i \notin A} c\left(s, p_{i}\right)+\sum_{B \cap A \neq \emptyset} c\left(q_{B}, t\right)=\sum_{i \notin A} c_{i}^{\prime}+\sum_{B \cap A \neq \emptyset} \alpha_{B} \\
& \geq \sum_{i \notin A} c_{i}^{\prime}+\sum_{i \in A} c_{i}^{\prime}=\sum_{i=1}^{n} c_{i}^{\prime} .
\end{aligned}
$$

By Claim $A$ and Theorem 5.3, there exists an $r$-s flow $f \leq c$ with value equal to $\sum_{i=1}^{n} c_{i}^{\prime}$. Notice that $f\left(s, p_{i}\right) \leq c_{i}^{\prime}$ but $\sum_{i=1}^{n} f\left(s, p_{i}\right)=\operatorname{value}(f)=$ $\sum_{i=1}^{n} c_{i}^{\prime}$, hence $f\left(s, p_{i}\right)=c_{i}^{\prime}$.

By the flow conservation law at the vertex $q_{A}$, for every $A \in \mathcal{P}_{n}$ we have

$$
\sum_{i \in A} f\left(p_{i}, q_{A}\right)=f\left(q_{A}, t\right) \leq c\left(q_{A}, t\right)=\alpha_{A},
$$

therefore we can choose numbers $\beta_{A, i}$ for $i \in A$ such that $\beta_{A, i} \geq f\left(p_{i}, q_{A}\right)$ and $\sum_{i \in A} \beta_{A, i}=\alpha_{A}$. We claim that these numbers have the desired property. To check this, we use again the flow conservation law, now at $p_{i}$ :

$$
c_{i}<c_{i}^{\prime}=f\left(s, p_{i}\right)=\sum_{A \ni i} f\left(p_{i}, q_{A}\right) \leq \sum_{A \ni i} \beta_{A, i} .
$$

\section{Fiber orders of the probability measures on a scattered com-} pact. As already mentioned, it is a standard fact that if $K$ is a totally disconnected compact and $\mathcal{B}$ is a basis for the topology of $K$ consisting of clopen sets, then a basis for the topology of $P(K)$ consists of the sets of the form $\left\{\mu \in P(K): \mu\left(U_{i}\right)>c_{i}, i=1, \ldots, n\right\}$, where the $c_{i}$ 's are positive numbers and the $U_{i}$ 's are disjoint basic clopen sets. When $K$ is scattered, all measures from $P(K)$ are discrete, and this allows us to find a finer neighborhood basis which will be quite useful for us. To avoid heavy notation, we write $\mu(x)$ instead of $\mu(\{x\})$ to denote the measure of a singleton.

Lemma 6.1. Let $K$ be a scattered compact space, $\mu \in P(K)$, and let $\mathcal{B}$ be a basis of the topology of $K$ consisting of clopen sets. A neighborhood basis of $\mu$ consists of the sets of the form $\left\{\nu: \nu\left(U_{i}\right)>c_{i}\right.$ for $\left.i=1, \ldots, n\right\}$, where $U_{1}, \ldots, U_{n}$ are pairwise disjoint basic clopen neighborhoods of points $x_{1}, \ldots, x_{n}$ of $K$ and $c_{1}, \ldots, c_{n}$ are positive numbers with $\mu\left(x_{i}\right)>c_{i}$. 
Proof. Consider a neigborhood of $\mu$ of the form $V=\{\nu \in P(K)$ : $\left.\nu\left(V_{j}\right)>d_{j}, j=1, \ldots, n\right\}$ for some disjoint basic clopen neighborhoods $V_{j}$ with $\mu\left(V_{j}\right)>d_{j}$. Since $\mu$ is discrete, for every $j$ we can find a finite family $\left\{x_{i}^{j}: i \in F_{j}\right\}$ of points such that $\sum\left\{\mu\left(x_{i}^{j}\right): i \in F_{j}\right\}>d_{j}$. Consider numbers $d_{i}^{j}<\mu\left(x_{i}^{j}\right)$ such that $\sum_{i \in F_{j}} d_{i}^{j}>d_{j}$, and disjoint basic clopen sets $U_{i}^{j}$ with $x_{i}^{j} \in U_{i}^{j} \subset V_{j}$. Then

$$
\mu \in\left\{\nu \in P(K): \nu\left(U_{i}^{j}\right)>d_{i}^{j}, j=1, \ldots, n, i \in F_{j}\right\} \subset V .
$$

For the rest of the section, we fix $g: K \rightarrow L$ to be a surjection between scattered compact spaces and $f=P(g): P(K) \rightarrow P(L)$ the induced map between the spaces of probability measures. Note that the norm we use below is the $\ell_{1}$-norm, i.e.

$$
\|\mu-\nu\|=\sum_{k \in K}|\mu(k)-\nu(k)| \quad \text { for } \mu, \nu \in P(K) .
$$

TheOREM 6.2. Let $\mu=\sum_{i \in I} r_{i} \delta_{x_{i}}$ be a probability measure on $L$, where $I=\mathbb{N}$ or $I=\{1, \ldots, N\}$ for some $N \in \mathbb{N}, x_{i}, i \in I$, are pairwise distinct points in $L$, and $r_{i}>0$ for all $i \in I$. Then the natural bijection $\prod_{i \in I} f^{-1}\left(\delta_{x_{i}}\right) \rightarrow f^{-1}(\mu)$ given by $\left(\nu_{i}\right)_{i \in I} \mapsto \sum_{i \in I} r_{i} \nu_{i}$ is an order-isomorphism. In particular, $\mathbb{F}_{\mu}(f) \cong \prod_{i \in I} \mathbb{F}_{\delta_{x_{i}}}(f)$ and $\mathbb{O}_{\mu}(f) \cong \prod_{i \in I} \mathbb{O}_{\delta_{x_{i}}}(f)$.

Proof. Consider the mapping $\Phi: P(K)^{I} \rightarrow P(K)$ defined by

$$
\Phi\left(\left(\nu_{i}\right)_{i \in I}\right)=\sum_{i \in I} r_{i} \nu_{i}
$$

It is easy to check that $\Phi$ is a continuous surjection. Moreover, as it is affine, it maps $\prod_{i \in I} f^{-1}\left(\delta_{x_{i}}\right)$ bijectively onto $f^{-1}(\mu)$. We will show that the restriction of $\Phi$ to $\prod_{i \in I} f^{-1}\left(\delta_{x_{i}}\right)$ is an order-isomorphism.

First, suppose that $\sum_{i \in I} r_{i} \nu_{i} \leq \sum_{i \in I} r_{i} \nu_{i}^{\prime}$ with $\nu_{i}, \nu_{i}^{\prime} \in f^{-1}\left(\delta_{x_{i}}\right)$ for $i \in I$; we shall prove that $\nu_{1} \leq \nu_{1}^{\prime}$. We consider a typical neighborhood of $\nu_{1}$ of the form

$$
U=\left\{\nu \in P(K): \nu\left(U_{j}\right)>c_{j} \text { for } j=1, \ldots, n\right\}
$$

where each $U_{j}$ is a clopen neighborhood of some $a_{j}$ satisfying $\nu_{1}\left(a_{j}\right)>c_{j}$ and $U_{j}$ 's are pairwise disjoint. We want to find a neighborhood $V$ of $\nu_{1}^{\prime}$ such that $f(V) \subset f(U)$.

We consider a very small number $\varepsilon>0$ such that

$$
\varepsilon<r_{1} \quad \text { and } \quad\left(r_{1}+\varepsilon\right)\left(c_{j}+2 \varepsilon\right)<r_{1} \nu_{1}\left(a_{j}\right) \text { for } j=1, \ldots, n .
$$

Choose $k \in I$ such that

$$
\sum_{i>k} r_{i}<\frac{1}{4} r_{1} \varepsilon\left(r_{1}-\varepsilon\right)
$$


and $H$ a clopen subset of $L$ such that $x_{1} \in H$ but $x_{i} \notin H$ for $2 \leq i \leq k$. The following is a neighborhood of $\sum_{i \in I} r_{i} \nu_{i}$ :

$$
U^{0}=\left\{\nu \in P(K): \nu\left(U_{j} \cap g^{-1}(H)\right)>\left(r_{1}+\varepsilon\right)\left(c_{j}+2 \varepsilon\right) \text { for } j=1, \ldots, n\right\} .
$$

By our assumption, there exists a neighborhood $V^{0}$ of $\sum_{i \in I} r_{i} \nu_{i}^{\prime}$ such that $f\left(V^{0}\right) \subset f\left(U^{0}\right)$. We take $V^{0}$ to be of the typical form

$$
V^{0}=\left\{\nu \in P(K): \nu\left(V_{j}\right)>d_{j} \text { for } j \in J\right\}
$$

where $J$ is a finite set, $V_{j}$ is a clopen neighborhood of some $b_{j}$ satisfying

$$
\sum_{i \in I} r_{i} \nu_{i}^{\prime}\left(b_{j}\right)>d_{j}
$$

and $V_{j}$ 's are pairwise disjoint. We let

$$
J_{i}=\left\{j \in J: g\left(b_{j}\right)=x_{i}\right\} .
$$

Without loss of generality we suppose that $V_{j} \subset g^{-1}(H)$ for $j \in J_{1}$, and $V_{j} \cap g^{-1}(H)=\emptyset$ for $j \in J_{2} \cup \cdots \cup J_{k}$. Notice that

$$
\sum\left\{d_{j}: j \in \bigcup_{i>k} J_{i}\right\}<\frac{1}{4} r_{1} \varepsilon\left(r_{1}-\varepsilon\right) .
$$

Consider now

$$
\begin{aligned}
V=\left\{\nu \in P(K): \nu\left(V_{j}\right)>d_{j} / r_{1} \text { for } j \in J_{1}\right\} \\
\\
\cap\left\{\nu \in P(K): \nu\left(K \backslash g^{-1}(H)\right)<\varepsilon / 2\right\} .
\end{aligned}
$$

This is a neighborhood of $\nu_{1}^{\prime}$ (notice that $\nu_{1}^{\prime}\left(K \backslash g^{-1}(H)\right)=0$ and $\nu_{1}^{\prime}\left(V_{j}\right) \geq$ $\nu_{1}^{\prime}\left(b_{j}\right)>d_{j} / r_{1}$ for $\left.j \in J_{1}\right)$. We claim that $f(V) \subset f(U)$.

So take $\xi_{1} \in V$. We can easily find $\xi_{2} \in V$ with $\left\|\xi_{2}-\xi_{1}\right\|<\varepsilon$ such that $\xi_{2}\left(K \backslash g^{-1}(H)\right)=0$. We pick a measure $\lambda \in P(K)$ with $\lambda\left(g^{-1}(H)\right)=0$ and $\lambda\left(V_{j}\right)>d_{j} /\left(1-r_{1}\right)$ for $j \in J_{2} \cup \cdots \cup J_{k}$. Then the measure $\xi_{3}=r_{1} \xi_{2}+\left(1-r_{1}\right) \lambda$ satisfies $\xi_{3}\left(V_{j}\right)>d_{j}$ for $j \in J_{1} \cup \cdots \cup J_{k}$. By (6.1) we may find $\xi_{4} \in V^{0}$ such that

$$
\left\|\xi_{4}-\xi_{3}\right\|<\frac{1}{2} r_{1} \varepsilon\left(r_{1}-\varepsilon\right)
$$

Set

$$
r=\xi_{4}\left(g^{-1}(H)\right) \quad \text { and } \quad \xi_{5}=\frac{1}{r} \xi_{4 \mid g^{-1}(H)} .
$$

We have

$$
\left|r_{1}-r\right|=\left|\xi_{3}\left(g^{-1}(H)\right)-\xi_{4}\left(g^{-1}(H)\right)\right|<\frac{1}{2} r_{1} \varepsilon\left(r_{1}-\varepsilon\right)<\varepsilon
$$


and

$$
\begin{aligned}
\left\|\xi_{2}-\xi_{5}\right\| & =\sum_{t \in g^{-1}(H)}\left|\xi_{2}(t)-\xi_{5}(t)\right|=\sum_{t \in g^{-1}(H)}\left|\frac{\xi_{3}(t)}{r_{1}}-\frac{\xi_{4}(t)}{r}\right| \\
& =\sum_{t \in g^{-1}(H)} \frac{1}{r_{1} r}\left|r \xi_{3}(t)-r_{1} \xi_{4}(t)\right| \leq \frac{1}{r_{1} r}\left\|r \xi_{3}-r_{1} \xi_{4}\right\| \\
& \leq \frac{1}{r_{1} r}\left\|\left(r_{1}-r\right) \xi_{4}\right\|+\frac{1}{r_{1} r}\left\|r \xi_{3}-r \xi_{4}\right\| \\
& \leq \frac{1}{r_{1} r}\left|r_{1}-r\right|+\frac{1}{r_{1}}\left\|\xi_{3}-\xi_{4}\right\| \\
& <\frac{\varepsilon\left(r_{1}-\varepsilon\right)}{2 r}+\frac{1}{2} \varepsilon\left(r_{1}-\varepsilon\right)<\frac{\varepsilon}{2}+\frac{\varepsilon}{2}=\varepsilon .
\end{aligned}
$$

The first inequality on the last line follows from the first inequality of (6.2), for the second one we use the fact that $r_{1}-\varepsilon<r$. It follows that $\left\|\xi_{1}-\xi_{5}\right\|<$ $2 \varepsilon$, and hence

$$
\left\|f\left(\xi_{1}\right)-f\left(\xi_{5}\right)\right\|<2 \varepsilon
$$

as well.

Now, $\xi_{4} \in V_{0}$, hence $f\left(\xi_{4}\right) \in f\left(V_{0}\right) \subset f\left(U_{0}\right)$. By the description of $f\left(U^{0}\right)$ given by Lemma 5.1, the fact that all clopen subsets of $K$ appearing in the definition of $U^{0}$ are contained in $g^{-1}(H)$ implies that

$$
\begin{gathered}
f\left(\xi_{5}\right) \in f\left(\left\{\nu \in P(K): \nu\left(U_{j} \cap g^{-1}(H)\right)>\left(r_{1}+\varepsilon\right)\left(c_{j}+2 \varepsilon\right) / r\right.\right. \\
\text { for } j=1, \ldots, n\}) \\
\subset f\left(\left\{\nu \in P(K): \nu\left(U_{j}\right)>c_{j}+2 \varepsilon \text { for } j=1, \ldots, n\right\}\right) .
\end{gathered}
$$

The inclusion above follows from (6.2); note that $r_{1}+\varepsilon>r$. Finally, using (6.4) and (6.3) it easily follows from Lemma 5.1 that $f\left(\xi_{1}\right) \in f(U)$, which completes the proof of the first implication.

We now pass to the converse. So we assume that $\nu_{i} \leq \nu_{i}^{\prime}$ for every $i$, and we shall see that $\sum_{i \in I} r_{i} \nu_{i} \leq \sum_{i \in I} r_{i} \nu_{i}^{\prime}$.

Let $U$ be a neighborhood of $\sum r_{i} \nu_{i}$ in $P(K)$. By the continuity of $\Phi$ and the definition of the product topology there is some $k \in I$ and neighborhoods $U_{i}$ of $\nu_{i}$ for $i \leq k$ such that

$$
\left\{\sum_{i \in I} r_{i} \lambda_{i}: \lambda_{i} \in P(K) \text { for } i \in I, \lambda_{i} \in U_{i} \text { for } i \leq k\right\} \subset U .
$$

As $\nu_{i} \leq \nu_{i}^{\prime}$ for all $i \in I$, there is, for each $i \leq k$, a neighborhood $V_{i}$ of $\nu_{i}^{\prime}$ such that $f\left(V_{i}\right) \subset f\left(U_{i}\right)$.

Now we are going to specify the form of $V_{i}$ 's. Let $H_{1}, \ldots, H_{k}$ be pairwise disjoint clopen subsets of $L$ containing $x_{1}, \ldots, x_{k}$, respectively. Then we can 
without loss of generality suppose that for each $i \leq k$ we have

$$
V_{i}=\left\{\lambda \in P(K): \lambda\left(V_{i}^{j}\right)>d_{i}^{j} \text { for } j \in J_{i}\right\}
$$

where $J_{i}$ is a finite set, $d_{i}^{j}>0$ for $j \in J_{i}$ and $V_{i}^{j}, j \in J_{i}$, are pairwise disjoint clopen subsets of $g^{-1}\left(H_{i}\right)$. Set

$$
V=\left\{\lambda \in P(K): \lambda\left(V_{i}^{j}\right)>r_{i} d_{i}^{j} \text { for } j \in J_{i}, i \leq k\right\} .
$$

Then $V$ is clearly a neighborhood of $\sum_{i \in I} r_{i} \nu_{i}^{\prime}$. We claim that $f(V) \subset f(U)$.

Let $\lambda \in V$ be arbitrary. Choose $\delta>0$ such that

$$
\begin{gathered}
(1+\delta) \sum_{j \in J_{i}} d_{i}^{j}<1, \quad i=1, \ldots, k ; \\
\lambda\left(V_{i}^{j}\right)>(1+\delta) r_{i} d_{i}^{j}, \quad j \in J_{i}, i=1, \ldots, k .
\end{gathered}
$$

Further, define the following measures:

$$
\begin{aligned}
\sigma_{i} & =\sum_{j \in J_{i}}(1+\delta) d_{i}^{j} \frac{\lambda_{\mid V_{i}^{j}}}{\lambda\left(V_{i}^{j}\right)}, \quad i=1, \ldots, k, \\
\tau & =\lambda-\sum_{i=1}^{k} r_{i} \sigma_{i} .
\end{aligned}
$$

All $\sigma_{i}$ 's are clearly positive measures. Moreover, $\tau$ is also positive by $(6.7)$, as

$$
\tau_{\mid V_{i}^{j}}=\lambda_{\mid V_{i}^{j}}\left(1-\frac{(1+\delta) r_{i} d_{i}^{j}}{\lambda\left(V_{i}^{j}\right)}\right)
$$

for $j \in J_{i}, i=1, \ldots, k$.

It follows from $(6.6)$ that $\sigma_{i}(K)<1$ for each $i=1, \ldots, k$, and so $\tau(K)>0$. For $i=1, \ldots, k$ set

$$
\theta_{i}=\sigma_{i}+\frac{1-\sigma_{i}(K)}{\tau(K)} \tau
$$

Then $\theta_{i} \in P(K)$. Moreover,

$$
\theta_{i}\left(V_{i}^{j}\right) \geq \sigma_{i}\left(V_{i}^{j}\right)=(1+\delta) d_{i}^{j}>d_{i}^{j}
$$

for $j \in J_{i}$, hence $\theta_{i} \in V_{i}$ for $i=1, \ldots, k$. We claim that

$$
\lambda \in\left\{\sum_{i \in I} r_{i} \lambda_{i}: \lambda_{i} \in V_{i} \text { for } i=1, \ldots, k\right\} .
$$

Indeed, we can take $\lambda_{i}=\theta_{i}$ for $i=1, \ldots, k$. To see this, we have to check that

$$
\vartheta=\lambda-\sum_{i=1}^{k} r_{i} \theta_{i}
$$


is a nonnegative measure:

$$
\begin{aligned}
\vartheta & =\lambda-\sum_{i=1}^{k} r_{i} \theta_{i}=\lambda-\sum_{i=1}^{k} r_{i}\left(\sigma_{i}+\frac{1-\sigma_{i}(K)}{\tau(K)} \tau\right) \\
& =\lambda-\sum_{i=1}^{k} r_{i} \sigma_{i}-\sum_{i=1}^{k} r_{i} \frac{1-\sigma_{i}(K)}{\tau(K)} \tau=\tau-\sum_{i=1}^{k} r_{i} \frac{1-\sigma_{i}(K)}{\tau(K)} \tau \\
& =\frac{\tau}{\tau(K)}\left(\tau(K)-\sum_{i=1}^{k} r_{i}+\sum_{i=1}^{k} r_{i} \sigma_{i}(K)\right)
\end{aligned}
$$

which is positive because $\sum_{i=1}^{k} r_{i} \leq 1=\lambda(K)=\tau(K)+\sum_{i=1}^{k} r_{i} \sigma_{i}(K)$. Thus, (6.8) is proved. Using (6.8) and (6.5) we see by Lemma 5.1 that $f(\lambda) \in f(U)$, which completes the proof.

Let $g: K \rightarrow L$ be a continuous surjection, $x \in L$ and $y_{1}, \ldots, y_{n} \in$ $g^{-1}(x)$. We define $\left\langle y_{1}, \ldots, y_{n}\right\rangle$ to be the set of all $z \in g^{-1}(x)$ such that for any neighborhoods $U_{1}, \ldots, U_{n}$ of $y_{1}, \ldots, y_{n}$ respectively there exists a neighborhood $V$ of $z$ such that $g(V) \subset g\left(U_{1}\right) \cup \cdots \cup g\left(U_{n}\right)$.

Notice some elementary properties, for instance $\langle y\rangle=\left\{z \in g^{-1}(x): z \geq y\right\}$ and $\langle Y\rangle \subset\left\langle Y^{\prime}\right\rangle$ whenever $Y \subset Y^{\prime}$. The $\langle\cdot\rangle$-operation provides in general a finer structure on the fiber $g^{-1}(x)$ than the one given by the order, and it is needed to determine the fiber order on spaces $P(K)$ in terms of the fibers of $K$. To avoid heavy notation, for a measure $\nu$, we often write $\nu\langle\cdot\rangle$ and $\nu\{\cdot\}$ instead of $\nu(\langle\cdot\rangle)$ and $\nu(\{\cdot\})$.

TheOREM 6.3. Let $\nu, \nu^{\prime}$ be elements of $f^{-1}\left(\delta_{x}\right)$. Then $\nu \leq \nu^{\prime}$ if and only if $\nu\left\langle y_{1}, \ldots, y_{n}\right\rangle \leq \nu^{\prime}\left\langle y_{1}, \ldots, y_{n}\right\rangle$ for any $y_{1}, \ldots, y_{n} \in g^{-1}(x)$.

Proof. Suppose first that $\nu \leq \nu^{\prime}$, and let $y_{1}, \ldots, y_{n} \in g^{-1}(x)$. If $\nu\left\langle y_{1}, \ldots, y_{n}\right\rangle$ $>\nu^{\prime}\left\langle y_{1}, \ldots, y_{n}\right\rangle$, this would mean that we can find $u_{1}, \ldots, u_{r} \in\left\langle y_{1}, \ldots, y_{n}\right\rangle$, and $v_{1}, \ldots, v_{s} \in g^{-1}(x) \backslash\left\langle y_{1}, \ldots, y_{n}\right\rangle$, and a number $\xi>0$ such that $\sum_{i=1}^{r} \nu\left(u_{i}\right)$ $>\xi$ and $\sum_{i=1}^{s} \nu^{\prime}\left(v_{i}\right)>1-\xi$. Since $v_{j} \notin\left\langle u_{1}, \ldots, u_{r}\right\rangle$ for any $j$ we can find neighborhoods $U_{i j}$ of $u_{i}$ such that $\bigcup_{i=1}^{r} g\left(U_{i j}\right)$ does not contain the $g$-image of any neighborhood of $v_{j}$. Set $U_{i}=\bigcap_{j=1}^{s} U_{i j}$. Consider a neighborhood of $\nu$ of the form

$$
U=\left\{\lambda \in P(K): \lambda\left(U_{i}\right)>d_{i}, i=1, \ldots, r\right\}
$$

where $d_{i}<\nu\left(u_{i}\right)$ and $\sum_{i=1}^{r} d_{i}>\xi$. We claim that $f(U)$ does not contain the image of any neighborhood of $\nu^{\prime}$, contradicting the fact that $\nu \leq \nu^{\prime}$. So take any neighborhood of $\nu^{\prime}$, which we can suppose of the normal form

$$
V=\left\{\lambda \in P(K): \lambda\left(V_{j}\right)>e_{j}, j=1, \ldots, k\right\}
$$

where the $V_{j}$ 's are disjoint neighborhoods of $w_{j}$ with $\nu^{\prime}\left(w_{j}\right)>e_{j}$ and moreover we can assume that $w_{j}=v_{j}$ for $j=1, \ldots, s$. For every $j=1, \ldots, s$ we 
can pick $x_{j} \in g\left(V_{j}\right) \backslash \bigcup_{i=1}^{r} g\left(U_{i}\right)$. Consider the measure $\lambda \in P(L)$ such that

$$
\lambda\left(x_{j}\right)=\sum\left\{\nu^{\prime}\left(v_{j^{\prime}}\right): x_{j^{\prime}}=x_{j}\right\} \quad \text { for } j=1, \ldots, s
$$

and $\lambda(x)=1-\sum_{j=1}^{s} \nu^{\prime}\left(v_{j}\right)$. Using Lemma 5.1 it easily follows that $\lambda \in f(V)$. Further, notice that

$$
\lambda\left(\bigcup_{i=1}^{r} g\left(U_{i}\right)\right) \leq 1-\sum_{i=1}^{s} \lambda\left(x_{i}\right)=1-\sum_{i=1}^{s} \nu^{\prime}\left(v_{i}\right)<\xi .
$$

This implies that $\lambda \notin f(U)$ because otherwise we should have

$$
\lambda\left(\bigcup_{i=1}^{r} g\left(U_{i}\right)\right)>\sum_{i=1}^{r} d_{i}>\xi .
$$

Now we suppose that $\nu\langle Y\rangle \leq \nu^{\prime}\langle Y\rangle$ for every finite set $Y \subset g^{-1}(x)$. We want to see that $\nu \leq \nu^{\prime}$ so we take a typical neighborhood of $\nu$ of the form

$$
U=\left\{\lambda \in P(K): \lambda\left(U_{i}\right)>c_{i}, i=1, \ldots, n\right\}
$$

where the $U_{i}$ 's are disjoint clopen neighborhoods of $y_{i}$ such that $\nu\left(y_{i}\right)>c_{i}$. For every nonempty $A \subset\{1, \ldots, n\}$ we have

$$
\nu^{\prime}\left\langle y_{i}: i \in A\right\rangle \geq \nu\left\langle y_{i}: i \in A\right\rangle \geq \nu\left\{y_{i}: i \in A\right\}>\sum_{i \in A} c_{i},
$$

and so there exists a finite set $\left\{z_{j}: j \in F_{A}\right\} \subset\left\langle y_{i}: i \in A\right\rangle$ such that

$$
\sum_{j \in F_{A}} \nu^{\prime}\left(z_{j}\right)>\sum_{i \in A} c_{i} .
$$

Pick $\xi_{j}<\nu^{\prime}\left(z_{j}\right)$ such that $\sum_{j \in F_{A}} \xi_{j}>\sum_{i \in A} c_{i}$. For every $j \in F_{A}$, since $z_{j} \in\left\langle y_{i}: i \in A\right\rangle$ we can find a clopen neighborhood $V_{j}$ of $z_{j}$ such that $g\left(V_{j}\right) \subset \bigcup_{i \in A} g\left(U_{i}\right)$. We can suppose that $V_{j} \cap V_{j^{\prime}}=\emptyset$ for different $j, j^{\prime} \in F_{A}$. Now, for every $A$ the following is a neighborhood of $\nu^{\prime}$ :

$$
V^{A}=\left\{\lambda \in P(K): \lambda\left(V_{j}\right)>\xi_{j}, j \in F_{A}\right\} .
$$

Let $V=\bigcap\left\{V^{A}: \emptyset \neq A \subset\{1, \ldots, n\}\right\}$. We claim that $f(V) \subset f(U)$. Take $\lambda \in f(V)$. According to Lemma 5.1 we have to check that for every nonempty $A \subset\{1, \ldots, n\}, \lambda\left(\bigcup_{i \in A} g\left(U_{i}\right)\right)>\sum_{i \in A} c_{i}$. Since $\lambda \in f(V) \subset f\left(V^{A}\right)$, by the same lemma we know that $\lambda\left(\bigcup_{j \in F_{A}} g\left(V_{j}\right)\right)>\sum_{j \in F_{A}} \xi_{j}>\sum_{i \in A} c_{i}$, and on the other hand $\bigcup_{j \in F_{A}} g\left(V_{j}\right) \subset \bigcup_{i \in A} g\left(U_{i}\right)$.

We finish this section by the following proposition which will enable us to verify the assumptions of Theorems 3.7 and 3.9 for spaces of the form $P(K)$.

Proposition 6.4. Let $K$ be a scattered compact space and $O$ a connected irreducible ordered set with $|O|>1$. 
(i) Suppose that there is a point $x \in K$ such that $\mathbb{O}_{\delta_{x}}(P(K)) \cong O$ and for each $y \in K \backslash\{x\}$ we have $\mathbb{O}_{\delta_{y}}(P(K)) \neq O^{k}$ for any $k \geq 1$. Then $P(K)$ satisfies the assumption of Theorem 3.7 .

(ii) Suppose that for the $\sigma$-typical surjection $f: L \rightarrow M$ (where $L$ and $M$ are metrizable quotients of $K$ ) there is $x \in M$ such that $\mathbb{O}_{\delta_{x}}(P(f)) \cong O, \mathbb{F}_{\delta_{x}}(P(f))$ has an equivalence class which is a singleton, and $\mathbb{F}_{\delta_{y}}(P(f))$ is a singleton for each $y \in M \backslash\{x\}$. Then $P(K)$ satisfies the assumptions of Theorem 3.9.

Proof. (i) We have $\mathbb{O}_{\delta_{x}}(P(K)) \cong O$. Further, suppose that $\mathbb{O}_{\mu}(P(K)) \cong O^{k}$ for some $k>1$ for some $\mu \in P(K)$. Let $C$ be a countable set supporting $\mu$. Then it follows from Theorem 6.2 and Corollary 3.6 that for the $\sigma$-typical surjection $f$ of $K$ there is some $y \in C \backslash\{x\}$ such that $\mathbb{O}_{\delta_{y}}(P(f)) \cong O^{j}$ for some $j \geq 1$. Now, as $C$ is countable, there is $y \in C^{*}=C \backslash\{x\}$ such that in each cofinal $\sigma$-semilattice in $K$ there is a surjection $f$ such that $\mathbb{O}_{\delta_{y}}(P(f)) \cong O^{j}$ for some $j \geq 1$, which contradicts our assumptions. (Otherwise, for every $y \in C^{*}$ there would be a cofinal $\sigma$-lattice $\mathcal{S}_{y} \subset \mathcal{Q}_{\omega}(K)$ with $\mathbb{O}_{\delta_{y}}(P(f)) \neq O^{j}$ for every $j$; an obvious improvement of Theorem 2.1 shows that $\bigcap_{y \in C^{*}} \mathcal{S}_{k}$ is a cofinal $\sigma$-semilattice, which leads to a contradiction). Thus we have verified the assumptions of Theorem 3.7.

(ii) Let $f: L \rightarrow M$ and $x \in M$ be as in the assumptions. Clearly $\mathbb{F}_{\delta_{y}}(P(f))$ is a singleton for each $y \in M \backslash\{x\}$. Therefore Theorem 6.2 shows that $\mathbb{O}_{\mu}(P(f)) \cong O$ if $\mu(x)>0$, and $\mathbb{F}_{\mu}(P(f))$ is a singleton if $\mu(x)=0$. In this way we have verified conditions (1)-(3) of Theorem 3.9. The remaining condition (4) follows immediately from Theorem 6.2.

\section{Examples of spaces of probability measures}

7.1. The space $\sigma_{n}(\kappa)$. In this section we are going to prove Theorem 1.4, the case of $P\left(\sigma_{n}(\kappa)\right)$ of Theorem 1.6 and the case of $P(A(\kappa))$ of Theorem 1.7.

For $N \subset M$, let $g_{M N}: \sigma_{n}(M) \rightarrow \sigma_{n}(N)$ be the continuous surjection given by $g=g_{N M}(x)=x \cap N$. The $\sigma$-typical surjection of $P\left(\sigma_{n}(\kappa)\right)$ is of the form $f=P(g): P\left(\sigma_{n}(M)\right) \rightarrow P\left(\sigma_{n}(N)\right)$ for $M \subset N$ infinite countable subsets of $\kappa$ such that $M^{*}=M \backslash N$ is infinite. We now compute the fiber order and the $\langle\cdot\rangle$-operation.

For $x \in \sigma_{n}(N), g^{-1}(x)=\left\{x \cup y: y \subset M^{*},|y| \leq n-|x|\right\}$. A basic neighborhood of such $x \cup y \in g^{-1}(x)$ is of the form

$$
U=\left\{z \in \sigma_{n}(M): x \cup y \subset z, z \cap u=\emptyset, z \cap v=\emptyset\right\},
$$

where $u \subset M^{*}$ and $v \subset N$ are finite sets. The image of such a neighborhood equals

$$
g(U)=\left\{z \in \sigma_{n}(N): x \subset z,|z| \leq n-|x \cup y|, z \cap v=\emptyset\right\} .
$$


From this it is clear that for $w, w^{\prime} \in g^{-1}(x)$ we have $w \leq w^{\prime}$ if and only if $|w| \leq\left|w^{\prime}\right|$, and also that

$$
\left\langle w_{1}, \ldots, w_{k}\right\rangle=\left\{w \in g^{-1}(x):|w| \geq \min \left(\left|w_{1}\right|, \ldots,\left|w_{k}\right|\right)\right\} .
$$

Thus, if we go to the spaces of probabilities, for $f=P(g): P\left(\sigma_{n}(M)\right) \rightarrow$ $P\left(\sigma_{n}(N)\right)$ and two measures $\nu, \nu^{\prime} \in f^{-1}\left(\delta_{x}\right)$, we have $\nu \leq \nu^{\prime}$ if and only if for every $k=1, \ldots, n-|x|$,

$$
\nu\left\{w \in g^{-1}(x):|w| \geq|x|+k\right\} \leq \nu^{\prime}\left\{w \in g^{-1}(x):|w| \geq|x|+k\right\} .
$$

Notice that $\langle x\rangle=g^{-1}(x)$, thus $\nu(\langle x\rangle)=\nu^{\prime}(\langle x\rangle)$ for all $\nu, \nu^{\prime} \in f^{-1}\left(\delta_{x}\right)$. The ordered set $\mathbb{O}_{\delta_{x}}(f)$ is thus isomorphic to

$$
\mathbb{O}_{\delta_{x}}(f) \cong\left\{t \in[0,1]^{n-|x|}: t_{1} \leq \cdots \leq t_{n-|x|}\right\} .
$$

Proposition 7.1. The ordered set $O_{k}=\left\{t \in[0,1]^{k}: t_{1} \leq \cdots \leq t_{k}\right\}$ is irreducible.

Proof. We proceed by induction on $k$. For $k=0, O_{0}$ is a singleton (by convention, if desired), and for $k=1, O_{1}=[0,1]$ is linearly ordered, so we suppose that $k \geq 2$ and that we have an order-isomorphism $\phi: O_{k} \rightarrow P \times Q$. We denote by 0 and 1 the minimum and maximum respectively of any of the ordered sets $O_{k}, P$ and $Q$ (all exist so that $\phi(0)=(0,0)$ and $\phi(1)=(1,1)$ ). Let

$$
\begin{aligned}
\Lambda & =\left\{t \in O_{k}: t_{2}=t_{3}=\cdots=t_{k}=1\right\} \\
& =\left\{t \in O_{k}:\{s: s \geq t\} \text { is linearly ordered }\right\} .
\end{aligned}
$$

Every element of $\phi(\Lambda)$ must be either of the form $(x, 1)$ or $(1, x)$, since otherwise $\{s: s \geq \phi(\lambda)\}$ cannot be linearly ordered. Moreover, since $\Lambda$ is linearly ordered, it follows that either $\phi(\Lambda) \subset P \times\{1\}$ or $\phi(\Lambda) \subset\{1\} \times Q$. We suppose that $\phi(\Lambda) \subset P \times\{1\}$. Now set $\lambda=(0,1, \ldots, 1) \in \Lambda$ and $\phi(\lambda)=$ $(u, 1)$. We have

$$
O_{k-1} \cong \phi\left\{t \in O_{k}: t \leq \lambda\right\}=\{s \in P: s \leq u\} \times Q,
$$

so by the inductive hypothesis, either $|Q|=1$ (which would finish the proof) or $u=0$. So we suppose that $u=0$, which implies that $Q \cong O_{k-1}$ and also $\phi(\Lambda)=P \times\{1\}$ (because we have found that $\phi(\lambda)=(0,1) \in \phi(\Lambda)$, and this is an upwards closed set). Thus $Q \cong O_{k-1}$ and $P \cong \Lambda \cong[0,1]$, and it remains to show that $O_{k} \neq O_{k-1} \times[0,1]$. The reason is that $p=((0,1, \ldots, 1), 1)$ and $q=((1, \ldots, 1), 0)$ are incomparable elements of $O_{k-1} \times[0,1]$ with the property that $\{t: t \geq p\}$ and $\{t: t \geq q\}$ are linearly ordered. However, we noticed that the set $\Lambda$ of points with this property in $O_{k}$ is linearly ordered.

Since one of our announced objectives was to show that $P\left(\sigma_{n}(\kappa)\right)$ is not homeomorphic to $P\left(\sigma_{m}(\kappa)\right)$ for $n \neq m$, let us now make it explicit why this is true. It is enough to notice that the irreducible ordered sets 
$O_{k}=\left\{t \in[0,1]^{k}: t_{1} \leq \cdots \leq t_{k}\right\}$ which appear in the fiber orders of these spaces are not order-isomorphic for different values of $k$, since for $n<m$, $O_{m}$ does not appear as the fiber order of any point of $P\left(\sigma_{n}(\kappa)\right)$. This can be realized in many different ways. For example, set

$$
e=(0,0, \ldots, 1)=\max \left\{t \in O_{k}:\{s: s \leq t\} \text { is linearly ordered }\right\} .
$$

Then $O_{k-1} \cong\left\{t \in O_{k}: t_{k}=1\right\}=\{t: t \geq e\}$. This shows that $O_{k-1}$ can be obtained in an intrinsic way from $O_{k}$, and thus if $O_{k} \cong O_{j}$, then $O_{k-1} \cong$ $O_{j-1}$. The inductive repetition of this argument leads to a contradiction if $k \neq j$.

Finally, let us show the appropriate parts of Theorems 1.6 and 1.7. We see easily that $\sigma_{n}(\kappa)$ satisfies the assumptions of Proposition 6.4(i) with $O=O_{n}$ and $x=\emptyset$. Further, $A(\kappa)=\sigma_{1}(\kappa)$ satisfies the assumptions of Proposition 6.4(ii) with $O=O_{1}=[0,1]$ and $x=\emptyset$.

7.2. The spaces $P\left(\left[0, \omega_{1}\right]^{n}\right)$ and $P\left(A(\kappa)^{n}\right)$. In this section we shall prove Theorem 1.5 and the appropriate part of Theorems 1.6 and 1.7.

The fiber orders of the two spaces of probability measures from the title can be computed in the same way. For $M \supset N$, let $p_{M N}: A(M) \rightarrow A(N)$ be the continuous surjection given by $p_{M N}(x)=x$ if $x \in A(N)$ and $p_{M N}(x)=$ $\infty$ otherwise. The $\sigma$-typical surjection of $P\left(A(\kappa)^{n}\right)$ is of the form $P\left(p_{M N}^{n}\right)$ : $P\left(A(M)^{n}\right) \rightarrow P\left(A(N)^{n}\right)$ for $M \supset N$ infinite countable subets of $\kappa$ such that $M \backslash N$ is infinite.

On the other hand, for countable ordinals $\alpha<\beta$ let $q_{\beta \alpha}:[0, \beta] \rightarrow[0, \alpha]$ be the continuous surjection given by $q_{\beta \alpha}(\gamma)=\gamma$ for $\gamma \leq \alpha$, and $q_{\beta \alpha}(\gamma)=\alpha$ for $\gamma>\alpha$. The $\sigma$-typical surjection of $P\left(\left[0, \omega_{1}\right]^{n}\right)$ is of the form $P\left(q_{\beta \alpha}^{n}\right)$ : $P\left([0, \beta]^{n}\right) \rightarrow P\left([0, \alpha]^{n}\right)$ where $\alpha<\beta$ are countable limit ordinals. From the point of view of fiber orders both surjections $p_{M N}$ and $q_{\beta \alpha}$ can be treated simultaneously since both can be viewed as a surjection $g: K \rightarrow L$ with the following properties:

( $\star$ There exist $\varpi \in L$ and $m \in g^{-1}(\varpi)$ such that $\left|g^{-1}(x)\right|=1$ for every $x \in L \backslash\{\varpi\}$, and with respect to the fiber order of $g^{-1}(\varpi)$, we have $m<t$ and $t \sim s$ for any $t, s \in g^{-1}(\varpi) \backslash\{m\}$.

In the case of $p_{M N}$ we should take $\varpi=\infty$ and $m=\infty$, while for $q_{\beta \alpha}$, $\varpi=\alpha$ and $m=\alpha$.

From now on, we concentrate on computing the fiber orders of $P\left(g^{n}\right)$ where $g: K \rightarrow L$ is a continuous surjection satisfying ( $\star$ ); the computation of the fiber orders of $P\left(A(\kappa)^{n}\right)$ and $P\left(\left[0, \omega_{1}\right]^{n}\right)$ will then follow immediately.

We fix $x=\left(x_{1} \ldots, x_{n}\right) \in L^{n}$, and set $R(x)=\left\{i \in\{1, \ldots, n\}: x_{i}=\varpi\right\}$. The first step is to determine the sets of the form $\left\langle y^{(1)}, \ldots, y^{(k)}\right\rangle$ in $\left(g^{n}\right)^{-1}(x)$. For every $y \in\left(g^{n}\right)^{-1}(x)$ we set $S(y)=\left\{i \in R(x): y_{i}>m\right\}$. 
Claim A. $\left\langle y^{(1)}, \ldots, y^{(k)}\right\rangle=\left\{z \in\left(g^{n}\right)^{-1}(x): \exists j \in\{1, \ldots, k\}: S(z) \supset\right.$ $\left.S\left(y^{(j)}\right)\right\}$.

Proof of Claim A. Suppose first that $S(z) \supset S\left(y^{(j)}\right)$ for some $j$. Then it follows immediately that $y^{(j)} \leq z$ since the inequality holds coordinatewise. Thus $z \in\left\langle y^{(1)}, \ldots, y^{(k)}\right\rangle$.

For the converse inclusion, suppose that for every $j$ there exists a coordinate $i(j) \in S\left(y^{(j)}\right) \backslash S(z)$. So $z_{i(j)}=m$ and $y_{i(j)}^{(j)}>m$. Since all the elements of $\mathbb{F}_{x}(g)$ which are greater than $m$ are equivalent, for every $y_{i}^{(j)}>m$ we can easily find a neighborhood $W_{i}^{j}$ such that $W=\bigcup_{i, j} g\left(W_{i}^{j}\right)$ does not contain the image of any neighborhood of $m$. For every $j \in\{1, \ldots, k\}$ set $U_{j}=\left\{y \in K^{n}: y_{i(j)} \in W_{i(j)}^{j}\right\}$, which is a neighborhood of $y^{(j)}$. We claim that $g^{n}\left(U_{1}\right) \cup \cdots \cup g^{n}\left(U_{k}\right)$ contains no image of a neighborhood of $z$, which will finish the proof of Claim A. Namely, if $V$ is a neighborhood of $z$ of the form $V_{1} \times \cdots \times V_{n}$ with $V_{i}$ a neighborhood of $z_{i}$, then for every $i \in R(x) \backslash S(z)$ we can find a point $t_{i} \in g\left(V_{i}\right) \backslash W$. If we take $u \in V$ with $g\left(u_{i}\right)=t_{i}$ for $i \in R(x) \backslash S(z)$, then $g^{n}(u) \notin g^{n}\left(U_{1}\right) \cup \cdots \cup g^{n}\left(U_{k}\right)$.

Claim B. For $\nu, \nu^{\prime} \in P\left(g^{n}\right)^{-1}\left(\delta_{x}\right)$, we have $\nu \leq \nu^{\prime}$ if and only if for every upwards closed subset $A \subset 2^{R(x)}$, we have $\nu\{z: S(z) \in A\} \leq \nu^{\prime}\{z: S(z) \in A\}$.

Proof of Claim B. It follows from Claim A that the subsets of $\left(g^{n}\right)^{-1}(x)$ of the form $\left\langle y^{(1)}, \ldots, y^{(k)}\right\rangle$ are exactly the sets of the form $\{z: S(z) \in A\}$ for some upwards closed family $A$ of subsets of $R(x)$.

As a consequence, for $x \in L^{n}$ with $|R(x)|=k$, we find that $\mathbb{O}_{\delta_{x}}\left(P\left(g^{n}\right)\right) \cong$ $\left\{t \in[0,1]^{2^{k}}: \sum_{i \in 2^{k}} t_{i}=1\right\}$, endowed with the order $t \leq s$ if and only if $\sum_{i \in A} t_{i} \leq \sum_{i \in A} s_{i}$ for every upwards closed subset of $2^{k}$.

Proposition 7.2. Consider the ordered set $P_{k}=\left\{t \in[0,1]^{2^{k}}: \sum_{i \in 2^{k}} t_{i}=1\right\}$ endowed with the order $t \leq s$ if and only if $\sum_{i \in A} t_{i} \leq \sum_{i \in A} s_{i}$ for every upwards closed subset of $2^{k}$. Then $P_{k}$ is an irreducible ordered set.

Proof. We proceed by induction on $k$. If $k=1$, then $P_{k} \cong[0,1]$. Suppose that we had an isomorphism $\phi: P_{k} \rightarrow Q \times R$. We use 0 and 1 to denote the minimum and maximum of any of these ordered sets (notice that that the minimum of $P_{k}$ is the characteristic function of the empty set $0=\chi_{\{\emptyset\}}$, and its maximum is $\left.1=\chi_{\{\{1, \ldots, n\}\}}\right)$. For every $i \in\{1, \ldots, n\}$ let $e^{i} \in P_{k}$ be the characteristic function of the singleton $\{\{i\}\}$. Notice that $\left\{t \in P_{k}: t \leq e^{i}\right\}$ is linearly ordered since any such $t$ satisfies $\sum_{a \not \subset\{i\}} t_{a}=0$. Thus $\phi\left(e^{i}\right)$ must be of the form either $\phi\left(e^{i}\right)=\left(u_{i}, 0\right)$ or $\phi\left(e^{i}\right)=\left(0, u_{i}\right)$. Notice now that

$$
\left\{t \in P_{k}: t \geq e^{i}\right\}=\left\{t \in P_{k}: \sum_{i \in a} t_{a}=1\right\} \cong P_{k-1}
$$


and $P_{k-1}$ is irreducible by the inductive hypothesis, so $u_{i}=1$ since $\{(r, s)$ : $\left.(r, s) \geq\left(r_{0}, s_{0}\right)\right\}=\left\{r: r \geq r_{0}\right\} \times\left\{s: s \geq s_{0}\right\}$. Hence $\phi\left(e^{i}\right) \in\{(1,0),(0,1)\}$ for every $i \in\{1, \ldots, k\}$. If $k>2$ this is already a contradiction, so we suppose that $k=2$ and $\phi\left(e^{1}\right)=(1,0)$ and $\phi\left(e^{2}\right)=(0,1)$. We denote the elements of $P_{2}$ by $t=\left(t_{\emptyset}, t_{\{1\}}, t_{\{2\}}, t_{\{1,2\}}\right)$. For every $\lambda \in[0,1]$, we set $x^{\lambda}=(1-\lambda, \lambda, 0,0)$ and $y^{\lambda}=(1-\lambda, 0, \lambda, 0)$ in $P_{2}$. We have $x^{\lambda} \leq e^{1}$ and $y^{\lambda} \leq e^{2}$ and so $\phi\left(x^{\lambda}\right)=\left(r^{\lambda}, 0\right)$ and $\phi\left(y^{\lambda}\right)=\left(0, s^{\lambda}\right)$ for suitable $r^{\lambda}$ and $s^{\lambda}$. We consider the specific elements $u=(0.7,0.1,0.1,0.1)$ and $u^{\prime}=(0.8,0,0,0.2)$ of $P_{2}$. Suppose $\phi(u)=(r, s)$ and $\phi\left(u^{\prime}\right)=\left(r^{\prime}, s^{\prime}\right)$. On the one hand, $x^{0.2}$ and $y^{0.2}$ are smaller than $u$ and $u^{\prime}$ so $r^{0.2} \leq r, r^{0.2} \leq r^{\prime}, s^{0.2} \leq s$ and $s^{0.2} \leq s^{\prime}$. On the other hand, if $\lambda>0.2$ then $x^{\lambda} \not \leq u, x^{\bar{\lambda}} \not{\leq} u^{\prime}, y^{\lambda} \not \leq u$, and $y^{\lambda} \not{Z} u^{\prime}$. Hence indeed $r=r^{\prime}=r^{0.2}$ and $s=s^{\prime}=s^{0.2}$. Thus $\phi(u)=\phi\left(u^{\prime}\right)$, a contradiction.

Notice that $P_{k}$ is not order-isomorphic to $P_{k^{\prime}}$ for $k \neq k^{\prime}$, since the set

$$
\begin{aligned}
H & =\left\{t \in P_{k}:\{s: s \leq t\} \text { is linearly ordered }\right\} \\
& =\left\{t \in P_{k}: \exists i \in\{1, \ldots, k\}: t \leq e^{i}\right\}
\end{aligned}
$$

contains exactly $k$ maximal elements: $e^{1}, \ldots, e^{k}$, where again $e^{i} \in P_{k}$ denotes the characteristic function of the singleton $\{i\}$. This also shows that these irreducible ordered sets are not isomorphic to the irreducible ordered sets $O_{k}$ which appeared in the fiber orders of the spaces $P\left(\sigma_{n}(\kappa)\right)$ (for $n>1$ ), because in those cases the set of all elements $t$ such that $\{s: s \leq t\}$ is linearly ordered was linearly ordered with precisely one maximal element.

The above calculation proves Theorem 1.5. Further, both $A(\kappa)^{n}$ and $\left[0, \omega_{1}\right]^{n}$ satisfy the assumptions of Proposition 6.4(i) with $O=P_{n}$ and $x=(\infty, \ldots, \infty)$ resp. $x=\left(\omega_{1}, \ldots, \omega_{1}\right)$. This proves the appropriate part of Theorem 1.6. Finally, $\left[0, \omega_{1}\right]$ satisfies the assumptions of Proposition 6.4(ii) with $O=[0,1]$ and $x=\alpha$ (using the above notation). This proves the appropriate part of Theorem 1.7.

We have not mentioned it so far, but despite the fact that the picture of fiber orders is similar, the spaces $P\left(A(\kappa)^{n}\right)$ and $P\left(\left[0, \omega_{1}\right]^{n}\right)$ are very different, for other well known reasons. Namely, $P\left(A(\kappa)^{n}\right)$ is an Eberlein compact, and hence a Fréchet-Urysohn space, so it cannot contain any copy of $\left[0, \omega_{1}\right]$.

8. Higher weights. So far we have used the version of spectral theorem stated as Theorem 2.1, but one can use other versions. For example, for a regular cardinal $\tau$, we consider the family $\mathcal{Q}_{\tau}(K)$ of quotients of weight strictly less than $\tau$, and we define a $\tau$-semilattice to be a subset $\mathcal{S} \subset \mathcal{Q}(K)$ such that the supremum of every subset of $\mathcal{S}$ of cardinality less than $\tau$ belongs to $\mathcal{S}$. The set $\mathcal{S}$ is cofinal in $\mathcal{Q}_{\tau}(K)$ if for every $L \in \mathcal{Q}_{\tau}(K)$ there exists $L^{\prime} \in \mathcal{S}$ with $L \leq L^{\prime}$. We assume that $\tau$ is a regular cardinal because otherwise there exists no cofinal $\tau$-semilattice in $\mathcal{Q}_{\tau}(K)$. 
THeOREM 8.1. Let $K$ be a compact space with weight at least $\tau$. The intersection of two cofinal $\tau$-semilattices in $\mathcal{Q}_{\tau}(K)$ is another cofinal $\tau$ semilattice in $\mathcal{Q}_{\tau}(K)$.

Proof. It is completely analogous to the proof of Theorem 2.1.

Lemma 8.2. Let $K$ be a compact space of weight at least $\tau$ and $\mathcal{S}$ a $\tau$ semilattice in $\mathcal{Q}_{\tau}(K)$. Then $\mathcal{S}$ is cofinal in $\mathcal{Q}_{\tau}(K)$ if and only if $\sup \mathcal{S}=K$.

Proof. Suppose $\sup \mathcal{S}=K$. By the same argument as in the proof of Lemma 2.2, every real-valued continuous function $f \in C(K)$ factors through an element of $\mathcal{S}$. Now, if $p: K \rightarrow L$ is an arbitrary element of $\mathcal{Q}_{\tau}(K)$, then we can take an embedding $L \subset \mathbb{R}^{\gamma}$ for a cardinal $\gamma<\tau$ and consider the functions $e_{i} p: K \rightarrow \mathbb{R}$ obtained by composing with the coordinate functions $e_{i}: \mathbb{R}^{\gamma} \rightarrow \mathbb{R}, i<\gamma$. For every $i<\gamma$ we know that there exists $L_{i} \in \mathcal{S}$ such that $e_{i} p$ factors through $L_{i}$. Finally, this implies that $p$ factors through $L_{\infty}=\sup \left\{L_{i}: i<\gamma\right\}$, so $L \leq L_{\infty} \in \mathcal{S}$.

Just as for $\sigma$-semilattice spectra, we can say that the $\tau$-typical surjection of $K$ has a property $\mathcal{P}$ if there is cofinal $\tau$-semilattice in which all the natural surjections have property $\mathcal{P}$, and when this happens such a $\tau$-semilattice can be found as a subsemilattice of any given one. Also similarly, we can talk in this context of $\mathbb{F}_{x}^{\tau}(K)$ and $\mathbb{O}_{x}^{\tau}(K)$.

An application can be found in the study of the space $P\left([0, \tau]^{n}\right)$ and its finite powers, for $\tau>\omega_{1}$ a regular cardinal. The fiber orders of $K=P\left([0, \alpha]^{n}\right)$ for any ordinal $\alpha \geq \omega_{1}$ can be computed using very similar arguments to those in Section 7.2, and indeed $\mathbb{O}_{\delta_{x}}(K) \cong P_{k}$ where $k$ is the number of coordinates of $x \in[0, \alpha]^{n}$ with uncountable cofinality. Therefore, for $\alpha \geq \omega_{1}+\omega_{1}$, $P\left([0, \alpha]^{n}\right)$ does not satisfy the assumptions of Proposition 6.4. Indeed, $[0, \alpha]$ has at least two non- $G_{\delta}$-points and hence $[0, \alpha]^{n}$ contains several points $x$ with $\mathbb{O}_{\delta_{x}}(P(K)) \cong P_{n}$. Moreover, $P\left([0, \alpha]^{n}\right)$ does not satisfy even the assumptions of Theorems 3.9 and 3.7 - for $n=1$ this is witnessed by the fact that $\mathbb{O}_{\frac{1}{2}\left(\delta_{x}+\delta_{y}\right)}(P([0, \alpha]))=[0,1]^{2}$ whenever $x$ and $y$ are two distinct points with uncountable cofinality.

However, it is still possible to get decomposition results for the spaces $P\left([0, \tau]^{n}\right)$ using $\tau$-semilattices, since analogues of Theorems 3.9 and 3.7 for the $\tau$-typical surjection hold, with identical proof. There is a natural cofinal $\tau$-semilattice for $[0, \tau]$ : For $\alpha<\beta$ consider the continuous surjection $p_{\beta \alpha}$ : $[0, \beta] \rightarrow[0, \alpha]$ given by $p_{\beta \alpha}(x)=x$ for $x \leq \alpha$, and $p_{\beta \alpha}(x)=\alpha$ for $x>\alpha$. The $\tau$-semilattice consists of all quotients given by $p_{\tau \alpha}, \alpha<\tau$, and the $\tau$-typical surjection is of the form $p_{\beta \alpha}, \alpha<\beta<\tau$. Thus, the situation is completely analogous to that of $P\left(\left[0, \omega_{1}\right]^{n}\right)$, and we have the following result:

THeOREM 8.3. Let $\tau$ be a regular cardinal, $K=P\left([0, \tau]^{n}\right), x=\left(x_{1}, \ldots, x_{r}\right)$ $\in[0, \tau]^{n}$ and $k=\left|\left\{i: x_{i}=\tau\right\}\right|$. Then $\mathbb{O}_{\delta_{x}}^{\tau}(K) \cong P_{k}=\left\{t \in[0,1]^{2^{k}}\right.$ : 
$\left.\sum_{i \in 2^{k}} t_{i}=1\right\}$ endowed with the order $t \leq s$ if and only if $\sum_{i \in A} t_{i} \leq \sum_{i \in A} s_{i}$ for every upwards closed subset of $2^{k}$.

Now we easily get the remaining part of Theorems 1.6 and 1.8.

We mention that, answering a question posed to us by R. Deville and G. Godefroy, the ideas of this section are used in [2] to show that there exist $2^{\kappa}$ many nonhomeomorphic weakly compact convex subsets in $\ell_{2}(\kappa)$.

\section{Final remarks and open problems}

Question 1. Let $M(K)$ denote the space of regular Borel measures of variation at most 1 (that is, the dual ball of the space of continuous functions $C(K))$ in its weak* topology. We show in this paper that $M(A(\kappa))$ is not homeomorphic to $P(A(\kappa))$ using fiber orders. We did not make a systematic study of the fiber orders of $M(K)$ and this may be interesting. Analysing the relatively easy case of $A(\kappa)$ it seems that the fiber orders of $M(A(\kappa))$ look similar to those of $P(A(\kappa))^{2}$, so we may ask whether $M(K) \approx P(K)^{2}$ for each compact space. However, this has a negative answer. Let $K$ be the wellknown "double arrow space". Then $P(K)$ is first countable (for example by [13, Proposition 7]) while $M(K)$ is not first countable as $K$ is not metrizable. Therefore $M(K) \not \approx P(K)^{2}$. But we can still ask: Is $M(K)$ homeomorphic to $P(K)^{2}$ for compact spaces considered in this paper $\left(A(\kappa), \sigma_{n}(\kappa)\right.$ etc. $)$ ?

Question 2. The analysis of the generic fibers of $B(\kappa)$ yields the same result as for $P(A(\kappa))$, namely all the non- $G_{\delta}$-points have generic fibers orderisomorphic to an interval. Are the spaces $B(\kappa)$ and $P(A(\kappa))$ homeomorphic? In this connection, it follows from [14] that $P(A(\kappa))$ is homeomorphic to $P(A(\kappa)) \times[0,1]$. Is $B(\kappa)$ homeomorphic to $B(\kappa) \times[0,1]$ or even to any product of two nontrivial spaces?

QUESTION 3. In the various spaces of probability measures that we have studied, fiber orders allow us to identify different types of points. Is this a complete classification? That is, we ask to determine exactly for which points $x, y \in P(K)$ there exists a homeomorphism $f: P(K) \rightarrow P(K)$ such that $f(x)=y$.

QUESTION 4. Fiber orders are a good tool to determine whether two spaces are homeomorphic, but they do not seem to help in determining whether a given space is the continuous image of another. In [3] the case of the spaces $B(\kappa)^{n}$ is studied, but the situation is not clear for the other spaces studied here. For instance, we do not know whether $P\left(\sigma_{n}(\kappa)\right)$ maps onto $P\left(\sigma_{m}(\kappa)\right)$ for $n<m$, and so on. This is also related to the problem of the $A(\kappa)^{\omega}$-images, initiated by Benyamini, Rudin and Wage [6] and studied specially by Bell in [4] and [5]. It is proven in [1] that $P(A(\kappa))$ and $B(\kappa)$ 
are continuous images of $A(\kappa)^{\omega}$, but it is unclear to us whether $P\left(\sigma_{n}(\kappa)\right)$ or $P\left(A(\kappa)^{n}\right)$ are continuous images of $A(\kappa)^{\omega}$ for $n>1$.

Acknowledgements. We want to express our gratitude to Stevo Todorčević for his valuable suggestions, and also to Richard Haydon, who proposed to us a shorter proof of Lemma 5.2. Thanks are also due to Petr Holický, Jiří Spurný and Miroslav Zelený for helpful comments.

The first author was supported by a Marie Curie Intra-European Fellowship MCEIF-CT2006-038768 (E.U.) and research projects MTM200508379 (MEC and FEDER) and Séneca 00690/PI/04.

The second author was supported by Research project MSM 0021620839 financed by MSMT and by Research grant GAČR 201/06/0018.

\section{References}

[1] A. Avilés, The unit ball of the Hilbert space in its weak topology, Proc. Amer. Math. Soc. 135 (2007), 833-836.

[2] - , The number of weakly compact convex sets which generate a Banach space, Topology Appl. 154 (2008), 404-409.

[3] - Compact spaces which do not map onto finite products, Fund. Math. 202 (2009), 81-96.

[4] M. Bell, A Ramsey theorem for polyadic spaces, ibid. 150 (1996), 189-195.

[5] —, Polyadic spaces of countable tightness, Topology Appl. 123 (2002), 401-407.

[6] Y. Benyamini, M. E. Rudin and M. Wage, Continuous images of weakly compact subsets of Banach spaces, Pacific J. Math. 70 (1977), 309-324.

[7] C. Bessaga and A. Pełczyński, Selected Topics in Infinite-Dimensional Topology, PWN-Polish Sci. Publ., Warszawa 1975.

[8] H. H. Corson and J. Lindenstrauss, On weakly compact subsets of Banach spaces, Proc. Amer. Math. Soc. 17 (1966), 407-412.

[9] G. B. Dantzig and D. R. Fulkerson, On the max-flow min-cut theorem of networks, in: Ann. of Math. Stud. 38, Princeton Univ. Press, 1956, 215-221.

[10] D. Fremlin, Splitting additive functionals, preprint, http://www.essex.ac.uk/maths/ staff/fremlin/preprints.htm.

[11] L. R. Ford Jr. and D. R. Fulkerson, Maximal flow through a network, Canad. J. Math. 8 (1956), 399-404.

[12] J. Hashimoto, On direct product decomposition of partially ordered sets, Ann. of Math. 54 (1951), 315-318.

[13] O. Kalenda, Few remarks on structure of certain spaces of measures, in: Continuitylike properties of mappings, Ph.D. thesis, Charles Univ. Prague, 1997, 46-57.

[14] -, On products with the unit interval, Topology Appl. 155 (2008), 1098-1104.

[15] A. Kechris, Classical Descriptive Set Theory, Grad. Texts in Math. 156, Springer, Berlin, 1994.

[16] J. Lindenstrauss, Weakly compact sets - Their topological properties and the Banach spaces that they generate, in: Symposium on Infinite-Dimensional Topology (Baton Rouge, LA, 1967), Ann. of Math. Stud. 69, Princeton Univ. Press, 1972, 235-273.

[17] J. van Mill, Infinite-Dimensional Topology. Prerequisites and Introduction, NorthHolland Math. Library 43, North-Holland, Amsterdam, 1989. 
[18] A. Schrijver, Combinatorial Optimization. Polyhedra and Efficiency, Algorithms Combin. 24, Springer, Berlin, 2003.

[19] E. V. Shchepin, Topology of limit spaces of uncountable inverse spectra, Russian Math. Surveys 31 (1976), no. 5, 155-191; transl. from Uspekhi Mat. Nauk 31 (1976), no. $5,191-226$.

[20] - Functors and uncountable powers of compacta, Russian Math. Surveys 36 (1981), no. 3, 1-71; transl. from Uspekhi Mat. Nauk 36 (1981), no. 3, 3-62.

[21] R. M. Schori, Hyperspaces and symmetric products of topological spaces, Fund. Math. 63 (1968), 77-88.

Facultad de Matemáticas

University of Murcia

Campus de Espinardo

Murcia, Spain

E-mail: avileslo@um.es
Department of Mathematical Analysis Faculty of Mathematics and Physics Charles University Sokolovská 83 18675 Praha 8, Czech Republic E-mail: kalenda@karlin.mff.cuni.cz

Received 21 April 2008;

in revised form 9 January 2009 